\title{
Exponential splines and minimal-support bases for curve representation $\sqrt{\mathrm{k}}$, 放出
}

\author{
R. Delgado-Gonzalo*, P. Thévenaz, M. Unser \\ Biomedical Imaging Group, École polytechnique fédérale de Lausanne (EPFL), Switzerland
}

\section{A R T I C L E I N F O}

\section{Article history:}

Received 9 September 2010

Received in revised form 7 October 2011

Accepted 26 October 2011

Available online 4 November 2011

\section{Keywords:}

Exponential B-spline

Exponential polynomial

Interpolation

Parameterization

Subdivision

Strang-Fix

Circular harmonics

\begin{abstract}
A B S T R A C T
Our interest is to characterize the spline-like integer-shift-invariant bases capable of reproducing exponential polynomial curves. We prove that any compact-support function that reproduces a subspace of the exponential polynomials can be expressed as the convolution of an exponential B-spline with a compact-support distribution. As a direct consequence of this factorization theorem, we show that the minimal-support basis functions of that subspace are linear combinations of derivatives of exponential Bsplines. These minimal-support basis functions form a natural multiscale hierarchy, which we utilize to design fast multiresolution algorithms and subdivision schemes for the representation of closed geometric curves. This makes them attractive from a computational point of view. Finally, we illustrate our scheme by constructing minimalsupport bases that reproduce ellipses and higher-order harmonic curves.
\end{abstract}

(C) 2011 Elsevier B.V. All rights reserved.

\section{Introduction}

The generation of curves under geometric restrictions is an important area of research in Computer Aided Geometric Design (CAGD). Considerable effort has been expended over the last forty years in this field in order to develop efficient and flexible representations of complex shapes. Since Bézier curves in the early sixties, B-spline curves in the mid seventies, and subdivision schemes in the late seventies, the search for representations that overcome the topological limitations of the classical approaches has not ceased. Research in this area has been fruitful and has resulted in many different methodologies (Böhm et al., 1984; Pham, 1992). They can be broadly categorized in terms of curve representation as

- subdivision schemes, where the curve is described as the limit of a refinement process (Micchelli and Prautzsch, 1989; Deslauriers and Dubuc, 1991; Warren and Weimer, 2002);

- parametric schemes, where the curve is described continuously by some coefficients using basis functions (Farin et al., 1987; Piegl, 1991; Farin, 1997; Schumaker, 2007).

A subdivision scheme is a set of rules that recursively define new points on finer grids starting form a set of initial points on a coarse grid. If the same rule is kept for all iterations, the scheme is called stationary (Cavaretta et al., 1991; Dyn et al., 1991; Dyn, 1992). If a different rule is used at each refinement level, the scheme is called nonstationary (Derfel et al., 1995;

\footnotetext{
this This work was funded by the Swiss SystemsX.ch initiative under Grant 2008/005 and the Swiss National Science Foundation under Grant 200020121763.

This paper has been recommended for acceptance by L.L. Schumaker.

* Corresponding author.

E-mail addresses: ricard.delgado@epfl.ch (R. Delgado-Gonzalo), philippe.thevenaz@epfl.ch (P. Thévenaz), michael.unser@epfl.ch (M. Unser).
} 
Dyn and Levin, 1995). Research is continually moving toward the investigation of refinement rules able to combine desirable reproduction properties under some geometrical constraints. In particular, schemes capable of reproducing circles were proposed in Zhang (1996), Zhang and Krause (2005), Sabin and Dodgson (2004), Deng and Wang (2010), Romani (2010), and, more recently, schemes based on exponential B-splines made possible the reproduction of conic sections (Beccari et al., 2007, 2009; Sunita and Shunmugaraj, 2009; Conti and Romani, 2010; Conti et al., 2011) and exponential polynomials (Dyn et al., 2008; Romani, 2009).

For certain applications, it is more convenient to represent the curve in an explicit parametric form instead of representing it as the limit of a subdivision process, the reason being that the parameters provide a direct way of evaluating any point on the curve. For computational reasons, short basis functions are preferable because the evaluation of a single point on the curve then depends on fewer coefficients.

In this paper, we are interested in designing a parametric curve representation model that can perfectly replicate ellipses as well as higher-order algebraic curves. To achieve this, we select basis functions that have the capability of reproducing specific families of exponential polynomials. We prove a factorization theorem that links the reproduction properties of a given basis function and its support. The theorem shows that any compact-support basis function that reproduces that subspace can be expressed as the convolution of an exponential B-spline and a compact-support distribution. As a corollary of this result, we obtain a full characterization of the minimal-support basis functions with the required reproduction properties; these basis functions were first identified by Ron using a different approach (Ron, 1990). This explicit characterization gives us the opportunity to identify interesting candidates within the family, and to construct nonstationary subdivision schemes that share the same reproduction properties.

The paper is organized as follows: In Section 2, we state the general parametric curve model through an expansion with compact-support basis functions, and discuss the requirements these bases should fulfill. In Section 3, we construct a family of basis functions that reproduce exponential polynomials and prove that these bases have minimal support. In Section 4 , we exhibit the multiresolution properties of our basis functions and propose a subdivision scheme that shares the same reproduction properties within the family. Finally, we illustrate the versatility of our model in Section 5 by identifying a basis from the family that contains ellipses and higher-order harmonics within its span.

\section{Parametric curves}

\subsection{Generic curves}

A curve $\mathbf{r}(t)$ on the plane can be described by a pair of Cartesian coordinate functions $x_{1}(t)$ and $x_{2}(t)$, where $t \in \mathbb{R}$ is a continuous parameter. We choose to parameterize the one-dimensional functions $x_{1}$ and $x_{2}$ by linear combinations of suitable basis functions. Among all possible bases, we focus on those derived from a compactly supported generator and its integer shifts $\{\varphi(\cdot-k)\}_{k \in \mathbb{Z}}$. This allows us to take advantage of fast and stable interpolation algorithms (Boehm, 1988; Costantini, 1988; Unser, 2000). The parametric representation of the curve is then given by the vectorial equation

$$
\mathbf{r}(t)=\sum_{k=-\infty}^{\infty} \mathbf{c}[k] \varphi\left(\frac{t}{T}-k\right)
$$

where $\{\mathbf{c}[k]\}_{k \in \mathbb{Z}}$ is a sequence of control points and $T$ a sampling step.

We want our parametric curve to be defined in terms of the coefficients in such a way that unicity of representation is satisfied. Furthermore, for computational purposes, we ask the interpolation procedure to be numerically stable. A generating function $\varphi$ is said to satisfy the Riesz-basis condition if and only if there exist two constants $0<A \leqslant B<\infty$ such that

$$
A\|\mathbf{c}\|_{\ell_{2}(\mathbb{Z})}^{2} \leqslant\left\|\sum_{k=-\infty}^{\infty} \mathbf{c}[k] \varphi(\cdot-k)\right\|_{L_{2}(\mathbb{R})}^{2} \leqslant B\|\mathbf{c}\|_{\ell_{2}(\mathbb{Z})}^{2},
$$

for all $\mathbf{c} \in \ell_{2}(\mathbb{Z})$. A direct consequence of the lower inequality is that the condition $\sum_{k=-\infty}^{\infty} \mathbf{c}[k] \varphi\left(\frac{t}{T}-k\right)=0$ for all $t \in \mathbb{R}$ implies that $\mathbf{c}[k]=0$ for all $k \in \mathbb{Z}$. Moreover, $\mathbf{c}[k]=0$ for all $k \in \mathbb{Z}$ trivially implies that $\sum_{k=-\infty}^{\infty} \mathbf{c}[k] \varphi\left(\frac{t}{T}-k\right)=0$ for all $t \in \mathbb{R}$. Therefore, the basis functions are linearly independent and every function is uniquely specified by its coefficients. Moreover, the upper inequality ensures the stability of the interpolation process (Unser, 2000; Aldroubi and Unser, 1994). Condition (2) can be expressed (Aldroubi and Unser, 1994) in the Fourier domain, where the following equivalent form must hold for every $\omega \in \mathbb{R}$ :

$$
A \leqslant \sum_{n=-\infty}^{\infty}|\hat{\varphi}(\omega+2 \pi n)|^{2} \leqslant B .
$$

The curve model in (1) has been shown to be very versatile since it can approximate any curve when the sampling step $T$ decreases while keeping the same basis function $\varphi$. The minimum requirement for this to happen is that $\varphi$ should be able to reproduce constants, which we formalize by 


$$
\sum_{k=-\infty}^{\infty} \varphi(\cdot-k)=1
$$

In the literature of approximation theory, this constraint is often named the partition-of-unity condition (de Boor and DeVore, 1985).

\subsection{Closed curves}

We are especially interested in the case when $\mathbf{r}$ is closed. In this context, the two coordinate functions are periodic, with same period. We normalize it to unity so that $\mathbf{r}(t)=\mathbf{r}(t+1)$ for all $t \in \mathbb{R}$, and divide it into $M$ segments, which is equivalent to choosing the sampling step $T=\frac{1}{M}$. Under these conditions, we can reduce the infinite summation in (1) to a finite one with $M$ terms involving periodized basis functions. We write

$$
\mathbf{r}(t)=\sum_{k=-\infty}^{\infty} \mathbf{c}[k] \varphi(M t-k)=\sum_{n=-\infty}^{\infty} \sum_{k=0}^{M-1} \mathbf{c}[M n+k] \varphi(M(t-n)-k)=\sum_{k=0}^{M-1} \mathbf{c}[k] \underbrace{\sum_{n=-\infty}^{\infty} \varphi(M(t-n)-k)}_{\varphi_{\text {per }}(M t-k)},
$$

where $M$ is the number of control points, the sequence $\{\mathbf{c}[k]\}_{k \in \mathbb{Z}}$ is $M$-periodic, and $\varphi_{\text {per }}$ is the $M$-periodization of the basis function $\varphi$. In the periodic setting, it has also been shown that this parametric curve model is very versatile (Jacob et al., 2002), and we can approximate any closed curve as accurately as we want by increasing the number of knots $M$. Under some mild refinability conditions, it has been shown that this model naturally leads to a stationary subdivision scheme (Micchelli and Prautzsch, 1989).

\subsection{Desirable properties of bases in the periodic settings}

We now enumerate the conditions that our parametric closed curve model should satisfy, and introduce the corresponding mathematical formalism.

1. Unique and stable representation. We want our closed parametric curve to be defined in terms of the coefficients in such a way that unicity of representation is satisfied, and we want the interpolation procedure to be numerically stable. A generating function $\varphi$ is said to satisfy the periodic Riesz-basis condition if and only if there exist two constants $0<A \leqslant B<\infty$ such that

$$
A\|\mathbf{c}\|_{\ell_{2}([0 \ldots M-1])}^{2} \leqslant\left\|\sum_{k=0}^{M-1} \mathbf{c}[k] \varphi_{\text {per }}(M \cdot-k)\right\|_{L_{2}([0,1])}^{2} \leqslant B\|\mathbf{c}\|_{\ell_{2}([0 \ldots M-1])}^{2}
$$

holds true for all $M$-periodic and bounded sequences $\mathbf{c}$. The interpretation of this condition is in all points similar to the non-periodic case. We also note that (5) is automatically satisfied if $\varphi_{\text {per }}$ is defined as in (4), and (2) holds true for $\varphi$.

2. Affine invariance. Since we are interested in representing shapes irrespective of their position and orientation, we would like our model to be invariant to affine transformations, which we formalize as

$$
\operatorname{Ar}(t)+\mathbf{b}=\sum_{k=0}^{M-1}(\mathbf{A c}[k]+\mathbf{b}) \varphi_{\mathrm{per}}(M t-k)
$$

where $\mathbf{A}$ is a $(2 \times 2)$ matrix and $\mathbf{b}$ is a two-dimensional vector. From (6), it is easy to show that the affine invariance is satisfied if and only if

$$
\sum_{k=0}^{M-1} \varphi_{\text {per }}(M \cdot-k)=1
$$

This last equality is a direct implication of the partition-of-unity condition (3).

\subsection{Approximation and reproduction properties in periodic settings}

The parametric closed-curve model (4) can be used to approximate any closed curve $\mathbf{s}$ as accurately as desired by increasing the number of knots $M$. Formally, we write that

$$
\lim _{M \rightarrow \infty}\left\|\mathbf{s}-\mathcal{P}_{M} \mathbf{s}\right\|_{L_{2}([0,1])}=0
$$


where $\mathcal{P}_{M} \mathbf{s}$ denotes a projection of $\mathbf{s}$ onto $\{\varphi(M \cdot-k)\}_{k \in \mathbb{Z}}$, or, equivalently, onto $\left\{\varphi_{\text {per }}(M \cdot-k)\right\}_{k=[0 \ldots M-1]}$, since both allow for alternative representations of the same space. In order to be able to select a suitable basis function, it is important to know the rate at which the error decreases as a function of $M$. The open-curve case reduces to the well-known Strang-andFix framework in approximation theory (Blu and Unser, 1999; Strang and Fix, 1971), the results of which are transposable to the closed-curve case as well (Jacob et al., 2002).

In addition to desirable approximation properties, our main interest lies in the situation where the curve $\mathbf{r}$ can reproduce desirable shapes exactly. For this purpose, we select for each $M \geqslant M_{0}$ a specific basis function capable of reproducing the

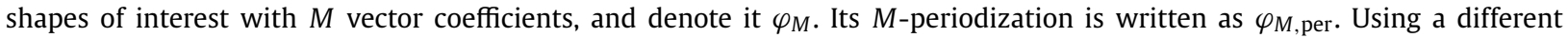
basis function $\varphi_{M}$ for each value of $M$ obviously leads to a subdivision scheme that is nonstationary. The existence of such a scheme depends on some refinability conditions over $\varphi_{M}$. In particular, the conditions of Section 2.3 have to hold for each $\varphi_{M}$ individually.

When the scheme is nonstationary, the approximation error of a curve $\mathbf{s}$ is $\left\|\mathbf{s}-\mathcal{P}_{M} \mathbf{s}\right\|_{L_{2}([0,1])}$, where now $\mathcal{P}_{M} \mathbf{s}$ denotes the projection of $\mathbf{s}$ onto $\left\{\varphi_{M}(N \cdot-k)\right\}_{k \in \mathbb{Z}}$, with $N=M$. Inspired by Dyn and Levin (1995), which discuss asymptotically equivalent binary subdivision schemes, we show in Section 5.1 .5 that the rate of decay of the approximation error as a function of $N=M$ is equivalent to that of the stationary case.

\section{Reproduction of exponential polynomials}

The main aim of this section is to introduce a family of functions that reproduce exponential polynomials, and prove that these functions have minimal support. To achieve this goal, we start by formalizing the concept of the reproduction of exponential polynomials. Next, we define the exponential B-splines and list their relevant properties. This allows us to give a full parameterization of the family of functions of interest: they happen to be combinations of exponential B-splines and their derivatives. Note that, in this section, we consider spline functions on a cardinal grid on the real line. The case of periodic spline functions corresponding to closed curves follows directly from this theory by the argument given in Section 2.3 , but the theory we develop here is more general and can also be used to design basis functions that reproduce non-periodic functions, for instance, open curves.

\subsection{Preliminary definitions}

A function $P_{\alpha}^{N}$ of the variable $t \in \mathbb{R}$ is called an exponential polynomial of degree $N$ and exponent $\alpha \in \mathbb{C}$ when it takes the form

$$
P_{\alpha}^{N}(t)=\mathrm{e}^{\alpha t}\left(a[0]+\sum_{n=1}^{N} a[n] t^{n}\right),
$$

where $\{a[n]\}_{n \in[0 \ldots N]}$ is a sequence of $(N+1)$ complex coefficients with $a[N] \neq 0$. A finite linear combination of exponential polynomials takes the form

$$
\sum_{m=1}^{M} p[m] P_{\alpha_{m}}^{N_{m}}
$$

A generating function $\varphi$ is said to reproduce a function $f$ if and only if there exists a sequence $\{c[k]\}_{k \in \mathbb{Z}}$ such that

$$
f(t)=\sum_{k=-\infty}^{\infty} c[k] \varphi(t-k)
$$

holds almost everywhere.

\subsection{Reproduction conditions}

A fundamental result in approximation theory is that there is an equivalence between the ability of a generating function to reproduce polynomials of a certain degree and the order of decay of the approximation error as the step size goes to zero (Unser and Blu, 2003). Strang and Fix (1971) showed that a generating function $\varphi \in L_{2}(\mathbb{R})$ has an approximation error that decays with order $N$ if and only if

$$
\int_{-\infty}^{\infty} \varphi(t) \mathrm{d} t \neq 0
$$

and there exists a finite constant $C_{n} \in \mathbb{C}$ such that 


$$
\sum_{k=-\infty}^{\infty}(t-k)^{n} \varphi(t-k)=C_{n}
$$

holds for almost every $t \in \mathbb{R}$, and for $n \in[0 \ldots N-1]$. Moreover, the generating function reproduces polynomials up to degree $(N-1)$.

An extension of the Strang-and-Fix conditions was presented by Vonesch et al. (2007) in the context of the reproduction of exponential polynomials. Here, we provide a reformulation suited to our needs.

Proposition 1. A compact-support generating function $\varphi \in L_{2}(\mathbb{R})$ reproduces exponential polynomials of degree up to $(N-1)$ and exponent $\alpha$ if and only if

$$
\int_{-\infty}^{\infty} \mathrm{e}^{-\alpha t} \varphi(t) \mathrm{d} t \neq 0
$$

and there exists a finite constant $C_{n} \in \mathbb{C}$ such that

$$
\sum_{k=-\infty}^{\infty}(t-k)^{n} \mathrm{e}^{-\alpha(t-k)} \varphi(t-k)=C_{n}
$$

holds for almost every $t \in \mathbb{R}$, and for $n \in[0 \ldots N-1]$.

This proposition is a direct consequence of the generalized Strang-and-Fix conditions from Vonesch et al. (2007) and the fact that $\varphi$ is compactly supported.

Another way of approaching the problem is offered in Unser and Blu (2005) where the authors show that the reproduction properties of generating functions are preserved through convolution. We summarize here their proposition for completeness.

Proposition 2. (See Unser and Blu, 2005.) Given a generating function $\varphi_{\alpha}$ that reproduces exponential polynomials of exponent $\alpha$ and degree up to $N$, then, for any $\psi$ such that $\int_{-\infty}^{\infty} \mathrm{e}^{-\alpha t} \psi(t) \mathrm{d} t \neq 0$, the composite function $\left(\varphi_{\alpha} * \psi\right)$ also reproduces exponential polynomials of exponent $\alpha$ and degree up to $N$.

Their formulation also requires two mild technical conditions over $\psi$ and $\left(\varphi_{\alpha} * \psi\right)$ to ensure that moments are welldefined.

Proposition 2 provides a constructive procedure to build generating functions using simpler functions with known reproduction properties. In the next section, we present the exponential B-splines, which will provide us with the appropriate building blocks to reproduce exponential polynomials.

\subsection{Exponential B-splines}

Exponential B-splines are the exponential counterpart of the well-known polynomial B-splines (Unser and Blu, 2005; Späth, 1969; de Boor and Ron, 1992). As their name suggests, they have the property of reproducing exponential polynomials, polynomials being recovered as a particular case by setting $\alpha=0$ in (8). An exponential B-spline of order $N$ and poles $\boldsymbol{\alpha}=\left(\alpha_{1}, \ldots, \alpha_{N}\right)$ is defined in the Fourier domain as

$$
\hat{\beta}_{\boldsymbol{\alpha}}(\omega)=\prod_{m=1}^{N} \frac{1-\mathrm{e}^{-\left(\mathrm{j} \omega-\alpha_{m}\right)}}{\mathrm{j} \omega-\alpha_{m}} .
$$

Note that the exponential B-splines are entirely specified by the collection $\boldsymbol{\alpha}$; the ordering of the poles $\alpha_{m}$ is irrelevant. We illustrate in Fig. 1 several exponential B-splines, where we see that a wide range of behaviors can be obtained by varying $N$ and $\boldsymbol{\alpha}$.

The most relevant properties of exponential B-splines for our purposes are

- The exponential B-splines are always well-defined (i.e., bounded and compactly supported), and form a Riesz basis if and only if $\left(\alpha_{m_{1}}-\alpha_{m_{2}}\right) \notin 2 \pi \mathrm{j} \mathbb{Z}$ for all pairs such that $m_{1} \neq m_{2}$.

- Exponential B-splines of order $N$ are compactly supported within the interval $[0, N]$.

- The convolution of two exponential B-splines yields another B-spline of augmented order

$$
\beta_{\boldsymbol{\alpha}_{1}} * \beta_{\boldsymbol{\alpha}_{2}}=\beta_{\boldsymbol{\alpha}_{1} \cup \boldsymbol{\alpha}_{2}}
$$

where $\left(\boldsymbol{\alpha}_{1} \cup \boldsymbol{\alpha}_{2}\right)$ is the concatenation of the elements of $\boldsymbol{\alpha}_{1}$ and $\boldsymbol{\alpha}_{2}$. 


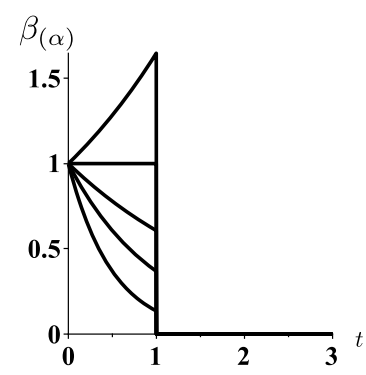

(a)

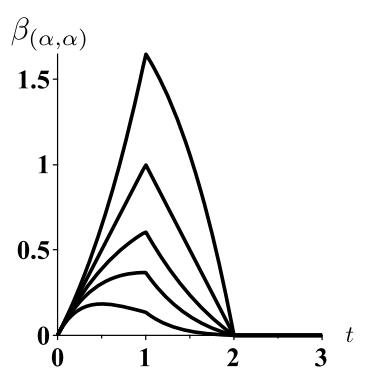

(b)

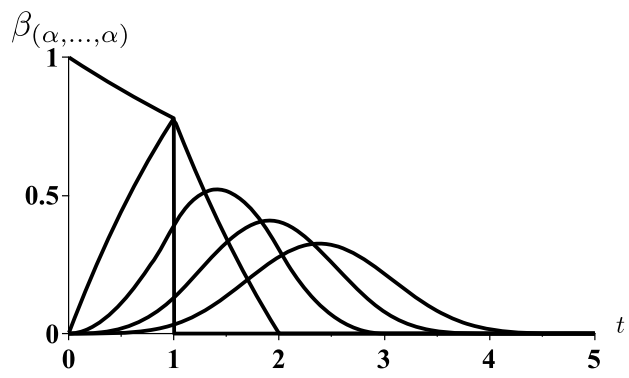

(c)

Fig. 1. Examples of exponential B-splines. (a) First-order exponential B-splines with $\alpha \in\left\{(-2),(-1),\left(-\frac{1}{2}\right),(0),\left(\frac{1}{2}\right)\right\}$. (b) Second-order exponential B-splines $\beta_{(\alpha, \alpha)}$ with $\alpha \in\left\{(-2,-2),(-1,-1),\left(-\frac{1}{2},-\frac{1}{2}\right),(0,0),\left(\frac{1}{2}, \frac{1}{2}\right)\right\}$. (c) Nth order exponential B-splines $\beta_{(\alpha, \ldots, \alpha)}$ with $\alpha=-\frac{1}{4}$ and $N \in[1 \ldots 5]$.

- The exponential B-splines of first order with parameter $(\alpha)$ reproduce the exponential function with exponent $\alpha$

$$
\mathrm{e}^{\alpha t}=\sum_{k=-\infty}^{\infty} \mathrm{e}^{\alpha k} \beta_{(\alpha)}(t-k) .
$$

- An exponential B-spline reproduces exponential polynomials of degree up to $\left(N_{m_{1}}-1\right)$ and exponent $\alpha_{m_{1}}$ if and only if $\alpha_{m_{1}}$ appears exactly $N_{m_{1}}$ times in $\boldsymbol{\alpha}$ and, for all other distinct $\alpha_{m_{2}}$, we have that $\left(\alpha_{m_{1}}-\alpha_{m_{2}}\right) \notin 2 \pi \mathrm{j} \mathbb{Z}$.

The three last properties provide us with a constructive procedure for building generating functions capable of reproducing exponential polynomials of a given degree and exponent. By construction, the support of the resulting generating functions corresponds to the order of the exponential B-spline. We refer to Unser and Blu (2005) for additional aspects of exponential B-splines.

\subsection{Distributional decomposition}

Our first goal is to characterize the functions that reproduce exponential polynomials. To that end, we are able to prove a converse version of Proposition 2; we prove that any compact-support function with the required reproduction properties must contain an exponential B-spline convolution factor with the same reproduction properties.

Theorem 1. Let $\varphi$ be supported within $[a, b]$ and let it reproduce finite linear combinations of exponential polynomials (9) such that $\left(\alpha_{m_{1}}-\alpha_{m_{2}}\right) \notin 2 \pi \mathrm{j} \mathbb{Z}$ for $m_{1} \neq m_{2}$. That is, $\varphi$ satisfies (10) and (11) for each pair $\left(N_{m}, \alpha_{m}\right)$. Then, a distribution $\psi$ exists such that

$$
\varphi=\beta_{\boldsymbol{\alpha}} * \psi,
$$

where $\psi$ satisfies (10) for all $\alpha_{m}$, each $\alpha_{m}$ appears $N_{m}$ times in $\boldsymbol{\alpha}$, and $\psi$ is compactly supported within $[a, b-N]$ with $N=\sum_{m=1}^{M} N_{m}$.

Proof. We proceed by induction over the order $N_{m_{1}}$ of each $\alpha_{m_{1}}$ to show that we can factor out $N_{m_{1}}$ times an exponential B-spline of first order for each $\alpha_{m_{1}}$ from the generating function $\varphi$. The process can be repeated for each exponent until the remaining kernel cannot reproduce any exponential polynomial anymore. Then, it is enough to show that, for a given $\alpha_{m_{2}}$, there exists a distribution $\psi$ such that

$$
\varphi=\beta_{\left(\alpha_{m_{2}}\right)} * \psi
$$

where $\psi$ satisfies the following properties:

1. it is compactly supported within $[a, b-1]$;

2. it reproduces exponential polynomials of degree up to $\left(N_{m_{2}}-2\right)$ and exponent $\alpha_{m_{2}}$;

3. it reproduces exponential polynomials of degree up to $\left(N_{m_{1}}-1\right)$ and exponent $\alpha_{m_{1}}$ for all $m_{1} \neq m_{2}$.

Since the definition of $\psi$ provided in (14) is implicit, we need to verify that this distributional kernel exists and is well-defined. We show this constructively. For a given $m_{2} \leqslant M$, we define the function

$$
\psi(t)=\sum_{k=0}^{\infty} \mathrm{e}^{\alpha_{m_{2}} k}\left(\mathrm{D}-\alpha_{m_{2}} \mathrm{I}\right) \varphi(t-k),
$$

where $\mathrm{D}$ is the derivative operator in the sense of distributions, and I is the identity. The infinite sum in (15) is well-defined since, for every $t$, the sum has only a finite number of elements because $\varphi$ has compact support. From (15), we write that 


$$
\psi(t)-\mathrm{e}^{\alpha_{m_{2}}} \psi(t-1)=\left(\mathrm{D}-\alpha_{m_{2}} \mathrm{I}\right) \varphi(t) .
$$

Taking the Fourier transform of (16) leads to the factorization

$$
\hat{\varphi}(\omega)=\frac{1-\mathrm{e}^{-\left(\mathrm{j} \omega-\alpha_{m_{2}}\right)}}{\mathrm{j} \omega-\alpha_{m_{2}}} \hat{\psi}(\omega)=\hat{\beta}_{\left(\alpha_{m_{2}}\right)}(\omega) \hat{\psi}(\omega)
$$

which corresponds to the implicit definition of $\psi$ given in (14).

To prove the first point, we recall that $\varphi$ reproduces exponential polynomials of degree up to $N_{m_{2}}-1 \geqslant 0$ and exponent $\alpha_{m_{2}}$. Thus, by setting $n=0$ in (11) and applying the differential operator ( $\left.\mathrm{D}-\alpha_{m_{2}} \mathrm{I}\right)$, we have that

$$
\sum_{k=-\infty}^{\infty} \mathrm{e}^{\alpha_{m_{2}}}\left(\mathrm{D}-\alpha_{m_{2}} \mathrm{I}\right) \varphi(t-k)=0
$$

in the distributional sense. Thanks to this last equality and using the explicit formula of $\psi$ given in (15), we can also write that

$$
\psi(t)=-\sum_{k=-\infty}^{-1} \mathrm{e}^{\alpha_{m_{2}} k}\left(\mathrm{D}-\alpha_{m_{2}} \mathrm{I}\right) \varphi(t-k) .
$$

According to this last expression, the support of $\psi$ is contained within $(-\infty, b-1]$. But, according to definition (15), we also have that the support of $\psi$ is contained within $[a,+\infty)$. Hence, we conclude that the support of $\psi$ is contained within $[a, b-1]$.

We deal with a modified version of (11) to prove the second point. By linearity, and since $\varphi$ reproduces exponential polynomials of degree up to $N_{m_{2}}-1 \geqslant 0$ and exponent $\alpha_{m_{2}}$, we can write that

$$
\sum_{k=-\infty}^{\infty} P(t-k) \mathrm{e}^{-\alpha_{m_{2}}(t-k)} \varphi(t-k)=C_{P}
$$

where $P$ is any polynomial of degree no greater than $\left(N_{m_{2}}-1\right)$, and $C_{P}$ is a constant that only depends on the polynomial $P$ and not on $t$. Then, the application of $\left(\mathrm{D}-\alpha_{m_{2}} \mathrm{I}\right)$ to (17) leads to

$$
0=\sum_{k=-\infty}^{\infty} P(t-k) \mathrm{e}^{-\alpha_{m_{2}}(t-k)} \underbrace{\left(\mathrm{D}-\alpha_{m_{2}} \mathrm{I}\right) \varphi(t-k)}_{\psi(t-k)-\mathrm{e}^{\alpha_{m_{2}} \psi(t-k-1)}}+\underbrace{\sum_{k=-\infty}^{\infty} \dot{P}(t-k) \mathrm{e}^{-\alpha_{m_{2}}(t-k)} \varphi(t-k),}_{C_{\dot{P}}}
$$

where we have used (16) to rewrite the first term, and where the second term is equal to the constant $C_{\dot{P}}$ since $\dot{P}$ is a polynomial of degree no greater than $\left(N_{m_{2}}-2\right)$. Since $\psi$ has a compact support, we can rearrange the terms as

$$
\sum_{k=-\infty}^{\infty} Q(t-k) \mathrm{e}^{-\alpha_{m_{2}}(t-k)} \psi(t-k)=-C_{\dot{P}}
$$

where $Q(t)=P(t)-P(t+1)$. Since $P$ is a polynomial of degree no greater than $\left(N_{m_{2}}-1\right)$, it follows that $Q$ is a polynomial of degree no greater than $\left(N_{m_{2}}-2\right)$. This also means that, for all polynomials $Q$ of degree no greater than $\left(N_{m_{2}}-2\right)$, there exists a constant $C_{Q}$ such that $\sum_{k=-\infty}^{\infty} Q(t-k) \mathrm{e}^{-\alpha_{m_{2}}(t-k)} \psi(t-k)=C_{Q}$. In particular, if $P(t)=t$, then $Q(t)=-1$. Because $\dot{P}$ is a polynomial of degree lesser than that of $P$, it also satisfies (17). Then, we can substitute $P$ by $\dot{P}=1$ in (17), which we combine with (18) and $Q=-1$ to obtain the system

$$
\left\{\begin{array}{l}
\sum_{k=-\infty}^{\infty} \mathrm{e}^{-\alpha_{m_{2}}(t-k)} \varphi(t-k)=C_{\dot{P}}, \\
-\sum_{k=-\infty}^{\infty} \mathrm{e}^{-\alpha_{m_{2}}(t-k)} \psi(t-k)=-C_{\dot{P}},
\end{array}\right.
$$

which leads to

$$
\sum_{k=-\infty}^{\infty} \mathrm{e}^{-\alpha_{m_{2}}(t-k)} \psi(t-k)=\sum_{k=-\infty}^{\infty} \mathrm{e}^{-\alpha_{m_{2}}(t-k)} \varphi(t-k) .
$$

Integrating the last expression of $t$ over the interval $[0,1]$, and rearranging the terms, yields 


$$
\int_{-\infty}^{\infty} \mathrm{e}^{-\alpha_{m_{2}} t} \psi(t) \mathrm{d} t=\int_{-\infty}^{\infty} \mathrm{e}^{-\alpha_{m_{2}} t} \varphi(t) \mathrm{d} t
$$

Thus, since $\varphi$ satisfies (10), so does $\psi$. Therefore, $\psi$ reproduces exponential polynomials of degree up to ( $N_{m_{2}}-2$ ) and exponent $\alpha_{m_{2}}$

Finally, to prove the third point, we proceed in the same manner. We recall that, for $m_{1} \neq m_{2}$, the function $\varphi$ reproduces exponential polynomials of degree up to $N_{m_{1}}-1 \geqslant 0$ and exponent $\alpha_{m_{1}}$. Thus, if we use (17) with parameter $\alpha_{m_{1}}$ and apply the differential operator $\left(\mathrm{D}-\alpha_{m_{2}} \mathrm{I}\right)$, then we obtain

$$
\left(\alpha_{m_{1}}-\alpha_{m_{2}}\right) C_{P}=\sum_{k=-\infty}^{\infty} P(t-k) \mathrm{e}^{-\alpha_{m_{1}}(t-k)} \underbrace{\left(\mathrm{D}-\alpha_{m_{2}} \mathrm{I}\right) \varphi(t-k)}_{\psi(t-k)-\mathrm{e}^{\alpha_{m_{2}}} \psi(t-k-1)}+\underbrace{\sum_{k=-\infty}^{\infty} \dot{P}(t-k) \mathrm{e}^{-\alpha_{m_{1}}(t-k)} \varphi(t-k)}_{C_{\dot{P}}^{\prime}},
$$

where we have used again (16) to rewrite the first term, and where the second term is equal to the constant $C_{\dot{p}}^{\prime}$ since $\dot{P}$ is a polynomial of degree no greater than $\left(N_{m_{1}}-2\right)$. Since $\psi$ has compact support, we can rearrange the terms to obtain

$$
\sum_{k=-\infty}^{\infty} Q(t-k) \mathrm{e}^{-\alpha_{m_{1}}(t-k)} \psi(t-k)=\left(\alpha_{m_{1}}-\alpha_{m_{2}}\right) C_{P}-C_{\dot{P}}^{\prime},
$$

where $Q(t)=P(t)-\mathrm{e}^{\alpha_{m_{2}}-\alpha_{m_{1}}} P(t+1)$. Since $P$ is a polynomial of degree no greater than $\left(N_{m_{1}}-1\right)$, and since $\mathrm{e}^{\alpha_{m_{2}}-\alpha_{m_{1}} \neq 1}$, then $Q$ is a polynomial of degree $\left(N_{m_{1}}-1\right)$, too. This also means that, for all polynomials $Q$ of degree no greater than $\left(N_{m}-1\right)$, there exists a constant $C_{Q}$ such that $\sum_{k=-\infty}^{\infty} Q(t-k) \mathrm{e}^{-\alpha_{m_{1}}(t-k)} \psi(t-k)=C_{Q}$. In addition, we see that, if $P(t)=1$, then $Q(t)=1-\mathrm{e}^{\alpha_{m_{2}}-\alpha_{m_{1}}}$ and $C_{\dot{P}}^{\prime}=0$. Now, by setting $P(t)=1$ in (17) and $Q(t)=1-\mathrm{e}^{\alpha_{m_{2}}-\alpha_{m_{1}}}$ in (19), we have the system

$$
\left\{\begin{array}{l}
\sum_{k=-\infty}^{\infty} \mathrm{e}^{-\alpha_{m_{2}}(t-k)} \varphi(t-k)=C_{P}, \\
\sum_{k=-\infty}^{\infty}\left(1-\mathrm{e}^{\alpha_{m_{2}}-\alpha_{m_{1}}}\right) \mathrm{e}^{-\alpha_{m_{1}}(t-k)} \psi(t-k)=\left(\alpha_{m_{1}}-\alpha_{m_{2}}\right) C_{P},
\end{array}\right.
$$

which leads to

$$
\sum_{k=-\infty}^{\infty} \mathrm{e}^{-\alpha_{m_{1}}(t-k)} \psi(t-k)=\frac{\alpha_{m_{1}}-\alpha_{m_{2}}}{1-\mathrm{e}^{-\left(\alpha_{m_{1}}-\alpha_{m_{2}}\right)}} \sum_{k=-\infty}^{\infty} \mathrm{e}^{-\alpha_{m_{2}}(t-k)} \varphi(t-k) .
$$

Integrating the last expression of $t$ over the interval $[0,1]$, and rearranging the terms, yields

$$
\int_{-\infty}^{\infty} \mathrm{e}^{-\alpha_{m_{1}} t} \psi(t) \mathrm{d} t=\frac{\alpha_{m_{1}}-\alpha_{m_{2}}}{1-\mathrm{e}^{-\left(\alpha_{m_{1}}-\alpha_{m_{2}}\right)}} \int_{-\infty}^{\infty} \mathrm{e}^{-\alpha_{m_{2}} t} \varphi(t) \mathrm{d} t
$$

Thus, since $\varphi$ satisfies (10) for $\alpha_{m_{2}}$, so does $\psi$ for $\alpha_{m_{1}}$. Therefore, $\psi$ reproduces exponential polynomials of degree up to $\left(N_{m_{1}}-1\right)$ and exponent $\alpha_{m_{1}}$.

\subsection{Minimal-support generating functions}

As a direct consequence of Theorem 1, we show that appropriate combinations of exponential B-splines define the whole family of functions of minimal support that reproduce exponential polynomials. This family was first identified in Ron (1990) by independent means.

Theorem 2. The size of the smallest-support kernel $\varphi \in L_{2}(\mathbb{R})$ that reproduces exponential polynomials of degree up to ( $\left.N_{m}-1\right)$ and parameter $\alpha_{m}$ for $m \in\{1 \ldots M\}$ is

$$
N=\sum_{m=1}^{M} N_{m}
$$

provided that $\left(\alpha_{m_{1}}-\alpha_{m_{2}}\right) \notin 2 \pi \mathrm{j} \mathbb{Z}$ for $m_{1} \neq m_{2}$. Moreover, every minimal-support function $\varphi$ can be written as 


$$
\varphi(t)=\sum_{n=0}^{N-1} \lambda_{n} \frac{\mathrm{d}^{n}}{\mathrm{~d} t^{n}} \beta_{\alpha}(t-a),
$$

where $a$ is an arbitrary shift parameter that determines the lower extremity of the support of $\varphi$. Moreover, each $\alpha_{m}$ appears exactly $N_{m}$ times within the collection $\alpha$ and the collection of $\lambda_{n}$ satisfies $\sum_{n=0}^{N-1} \lambda_{n} \alpha_{m}^{n} \neq 0$.

Proof. By Theorem 1, we can write

$$
\varphi=\beta_{\boldsymbol{\alpha}} * \psi
$$

where $\psi$ is a distribution with support $[a, b-N]$ that satisfies (10) for all $\alpha_{m}$. Finally, each $\alpha_{m}$ appears $N_{m}$ times within the collection $\boldsymbol{\alpha}$. Conversely, if we take a distribution $\psi$ that satisfies (10) for all $\alpha_{m}$ and is supported within [a, $b^{\prime}$ ], then $\varphi=\beta_{\boldsymbol{\alpha}} * \psi$ is supported within $\left[a, b^{\prime}+N\right]$ and reproduces exponential polynomials of degree up to $\left(N_{m}-1\right)$ and parameter $\alpha_{m}$ for $m \in[1 \ldots M]$. Now, minimizing the support of $\varphi$ means finding the smallest $b$ such that $\psi$ exists. Of course, this is possible only if $b^{\prime}=b-N \geqslant a$, which yields $\psi$ as a single-point distribution. This shows that the minimum size of the support of $\varphi$ is $b-a=N$.

We know from distribution theory that the only distributions that have a support of zero-measure are finite linear combinations of the Dirac distribution and of its derivatives (Schwarz, 1966, Th. XXXV). Thus, if $\varphi$ has minimal support, then there exist constants $\lambda_{n}$ such that

$$
\psi(t)=\sum_{n=0}^{\infty} \lambda_{n} \delta^{(n)}(t-a) .
$$

This means that

$$
\varphi(t)=\sum_{n=0}^{\infty} \lambda_{n} \frac{\mathrm{d}^{n}}{\mathrm{~d} t^{n}} \beta_{\alpha}(t-a) .
$$

Since we restrict ourselves to $L_{2}(\mathbb{R})$, the summation has to run from 0 to $(N-1)$.

Finally, since $\psi$ satisfies (10) for all $\alpha_{m}$, we have that

$$
\begin{array}{rlr}
0 & \neq \int_{-\infty}^{\infty} \mathrm{e}^{-\alpha_{m} t} \psi(t) \mathrm{d} t & \text { by hypothesis } \\
& =\int_{-\infty}^{\infty} \mathrm{e}^{-\alpha_{m} t} \sum_{n=0}^{N-1} \lambda_{n} \delta^{(n)}(t) \mathrm{d} t & \text { by }(22) \\
& =\sum_{n=0}^{N-1} \lambda_{n} \int_{-\infty}^{\infty} \mathrm{e}^{-\alpha_{m} t} \delta^{(n)}(t) \mathrm{d} t & \text { by linearity } \\
& =\sum_{n=0}^{N-1} \lambda_{n}\left\langle\delta^{(n)}(t), \mathrm{e}^{-\alpha_{m} t}\right\rangle & \text { by definition } \\
& =\sum_{n=0}^{N-1} \lambda_{n} \alpha_{m}^{n},
\end{array}
$$

which proves the last result.

\subsection{Interpolator}

It is also possible to constrain $\varphi$ to be an interpolator. That is,

$$
\forall k \in \mathbb{Z}:\left.\quad \varphi(t)\right|_{t=k}=\delta[k] .
$$

Due to the size of the support of $\varphi$, the interpolation condition can add up to $N$ constraints, depending on the value of $a$. This number of constraints matches the $N$ degrees of freedom that result from the choice of $\lambda_{n}$ in (21). A general study of the appropriate choice of $\lambda_{n}$ to satisfy the interpolation condition lies out of the scope of this paper. However, we propose a case-by-case approach that will be exemplified in Section 5. 


\section{Multiresolution and subdivision}

We have characterized the complete family of functions with minimal support that reproduce exponential polynomials in order to build parametric curves. In this section, we emphasize the connection with the subdivision world using the classical multiresolution properties of exponential B-splines. Moreover, we also specify another type of multiresolution scheme in terms of reproduction capabilities. In this section we focus on our case of interest: closed curves.

\subsection{Classical multiresolution of exponential B-splines}

An important observation concerning the family of minimal-support basis functions in (21) is that it is constructed with exponential B-splines and their derivatives of equal parameter $\boldsymbol{\alpha}$. Thanks to this property and under appropriate circumstances, the basis functions in (21) inherit the multiresolution properties of the exponential B-splines. It has been shown in Unser and Blu (2005), de Boor et al. (1993) that an exponential B-spline and its derivatives with parameter $\boldsymbol{\alpha}=\left(\alpha_{1}, \ldots, \alpha_{N}\right)$ satisfy the nonstationary set of dilation relations

$$
\begin{aligned}
& \beta_{\alpha}\left(\frac{t}{2}\right)=\sum_{k=-\infty}^{\infty} h_{\frac{\alpha}{2}}[k] \beta_{\frac{\alpha}{2}}(t-k), \\
& \frac{\mathrm{d}}{\mathrm{d} t} \beta_{\alpha}\left(\frac{t}{2}\right)=2 \sum_{k=-\infty}^{\infty} h_{\frac{\alpha}{2}}[k] \frac{\mathrm{d}}{\mathrm{d} t} \beta_{\frac{\alpha}{2}}(t-k), \\
& \vdots \\
& \frac{\mathrm{d}^{n}}{\mathrm{~d} t^{n}} \beta_{\alpha}\left(\frac{t}{2}\right)=2^{n} \sum_{k=-\infty}^{\infty} h_{\frac{\alpha}{2}}[k] \frac{\mathrm{d}^{n}}{\mathrm{~d} t^{n}} \beta_{\frac{\alpha}{2}}(t-k),
\end{aligned}
$$

where $n \leqslant(N-1), \frac{\alpha}{2}=\left(\frac{\alpha_{1}}{2}, \ldots, \frac{\alpha_{N}}{2}\right)$ is the collection of roots divided by 2 , and $h_{\frac{\alpha}{2}}$ is the mask whose symbol is given by

$$
H_{\frac{\alpha}{2}}(z)=\frac{1}{2^{N-1}} \prod_{m=1}^{N}\left(1+\mathrm{e}^{\frac{\alpha_{m}}{2}} z^{-1}\right) .
$$

\subsection{Subdivision scheme}

We have now all the ingredients in hand to define a multiresolution hierarchy of spaces of closed curves. We define the spline space at resolution $M$ as

$$
V_{\boldsymbol{\alpha}, M}=\left\{\mathbf{r}(t)=\sum_{k=0}^{M-1} \mathbf{c}_{M}[k] \varphi_{M, \operatorname{per}}(M t-k)\right\},
$$

where $M$ is the number of control points of the curve, and $\varphi_{M \text {,per }}$ is the $M$-periodization of (21) with defining parameter $\frac{\alpha}{M}$. Note that the set of parameters $\left\{\lambda_{n}\right\}_{n=0 \ldots N-1}$, which are used to define $\varphi_{M \text {,per }}$ through (21) and (4), depend on $M$. In order to find the equivalent scaling expression for our generating function $\varphi_{M}$, we proceed in the Fourier domain where the explicit expression of $\varphi_{M}$ in terms of exponential B-splines is

$$
\hat{\varphi}_{M}(\omega)=\Lambda_{M}(\mathrm{j} \omega) \hat{\beta}_{\frac{\alpha}{M}}(\omega) \mathrm{e}^{-\mathrm{j} \omega a} .
$$

There, the Fourier-domain function $\Lambda_{M}(\mathrm{j} \omega)=\lambda_{0}[M]+\sum_{n=1}^{N-1} \lambda_{n}[M](\mathrm{j} \omega)^{n}$ is a polynomial in (j $\omega$ ) of degree no greater than $(N-1)$. To derive the scaling relation, we take $\varphi_{M}\left(\frac{t}{2}\right)$ and $\varphi_{2 M}(t)$ to the Fourier domain. We have that

$$
\frac{2 \hat{\varphi}_{M}(2 \omega)}{\hat{\varphi}_{2 M}(\omega)}=\frac{2 \Lambda_{M}(\mathrm{j} 2 \omega) \hat{\beta}_{\frac{\alpha}{M}}(2 \omega) \mathrm{e}^{-\mathrm{j} 2 \omega a}}{\Lambda_{2 M}(\mathrm{j} \omega) \hat{\beta}_{\frac{\alpha}{2 M}}(\omega) \mathrm{e}^{-\mathrm{j} \omega a}} .
$$

By identifying the Fourier symbol $H_{\frac{\alpha}{2 M}}\left(\mathrm{e}^{\mathrm{j} \omega}\right)$ of the scaling relation, we can rewrite (25) as

$$
\frac{2 \hat{\varphi}_{M}(2 \omega)}{\hat{\varphi}_{2 M}(\omega)}=\frac{\Lambda_{M}(\mathrm{j} 2 \omega)}{\Lambda_{2 M}(\mathrm{j} \omega)} H_{\frac{\alpha}{2 M}}\left(\mathrm{e}^{\mathrm{j} \omega}\right) \mathrm{e}^{-\mathrm{j} \omega a} .
$$

Using this result, it is straightforward to verify that $V_{\boldsymbol{\alpha}, M} \subset V_{\boldsymbol{\alpha}, 2 M}$, provided that $a$ in $(21)$ is an integer and $\frac{\Lambda_{M}(\mathrm{j} 2 \omega)}{\Lambda_{2 M}(\mathrm{j} \omega)}$ is a $2 \pi$-periodic function. If $a$ is noninteger, a similar multiresolution embedding space scheme can be achieved by shifting the 
grid at each refinement level. In any case, the particular choice of the set of parameters $\left\{\lambda_{n}\right\}_{n=0 . . . N-1}$ will determine if the basis function is refinable and, therefore, if the multiresolution spaces are nested or not. We analyze in Section 5.1.3 how (26) applies to the various bases proposed in this paper. In addition, we illustrate in Section 5.1.4 a constructive procedure to determine a nontrivial set of $\left\{\lambda_{n}\right\}_{n=0 \ldots N-1}$ that satisfies (26) and generates refinable schemes.

In the case where the spaces are nested, the subdivision process for finding the sequence of coefficients $\mathbf{c}_{2 M}$ starting with the sequence $\mathbf{c}_{M}$ is then carried out in the following two steps:

1. upsampling of the original sequence $\mathbf{c}_{M}$ with a factor of 2 ;

2. filtering of the upsampled sequence with a smoothing filter $\tilde{h}_{\frac{\alpha}{2}}$ using periodic boundary conditions.

The filter $\tilde{h}_{\boldsymbol{\alpha}}$ will depend on the particular choice of the parameters $\left\{\lambda_{n}\right\}_{n=0 . . N-1}$, and its construction will be exemplified in Section 5 for the case of centered basis functions. The sequence $\mathbf{c}_{2 M}$ of $2 M$ coefficients represents exactly the same parametric curve as the original sequence $\mathbf{c}_{M}$ of $M$ coefficients. This process can be repeated indefinitely to obtain finer representations of the curve in a dyadic fashion.

\subsection{Multiresolution-reproduction capabilities}

An alternative multiresolution scheme emerges as we concatenate new elements to $\boldsymbol{\alpha}$ for fixed $M$. Since the reproduction of exponential polynomials is fully determined by $\boldsymbol{\alpha}$, the incorporation of additional elements does not perturb the reproduction capabilities. This multiresolution scheme in the reproduction properties will be exemplified in the case of multiple harmonics in Section 5.2.

\section{Applications}

In this section, we make use of Theorem 2 to build basis functions with minimal support capable of reproducing sinusoids. We start with single-frequency sinusoids that lead to ellipses, and then we derive the basis functions for generating higher-order harmonics.

\subsection{Reproduction of ellipses}

Circles and ellipses deserve a special attention since these simple shapes appear frequently in images in many fields, for example computer graphics and biomedical engineering. Since all ellipses can be obtained by applying an affine transformation to the unit circle, we focus on the reproduction of this simple shape. This allows us to take advantage of the requirement for affine invariance that we stated in Section 2.3.

A parametric curve defined by $M$ vectorial coefficients and by an $M$-dependent generating function $\varphi_{M}$ is said to reproduce the unit circle if there exist two $M$-periodic sequences $\left\{c_{c}[k]\right\}_{k \in \mathbb{Z}}$ and $\left\{c_{s}[k]\right\}_{k \in \mathbb{Z}}$ such that

$$
\begin{aligned}
& \cos (2 \pi t)=\sum_{k=0}^{M-1} c_{\mathrm{c}}[k] \varphi_{M, \mathrm{per}}(M t-k), \\
& \sin (2 \pi t)=\sum_{k=0}^{M-1} c_{\mathrm{S}}[k] \varphi_{M, \text { per }}(M t-k) .
\end{aligned}
$$

We illustrate in Fig. 2 the reproduction of sinusoids of unit period for each component. Note that, when (27) and (28) hold, it is possible to represent any sinusoid of unit period for an arbitrary initial phase using linear combinations of the two sequences of coefficients.

\subsubsection{Minimal-support basis for sinusoids with maximum smoothness}

We now particularize Theorem 2 for the case of sinusoids keeping the maximum degree of smoothness for $\varphi_{M}$. This particular case is of special interest to us. We were able to take advantage of it to build an efficient active contour capable of reproducing ellipses (Delgado-Gonzalo et al., 2011).

Corollary 1. The centered generating function with minimal support and maximal smoothness that satisfies all conditions in Section 2.3 and that reproduces sinusoids of unit period with $M$ coefficients is

$$
\varphi_{M}^{S}(t)=\sum_{k=0}^{3}(-1)^{k} c_{M}^{S}[k] \varsigma_{M}\left(t+\frac{3}{2}-k\right)
$$

where 


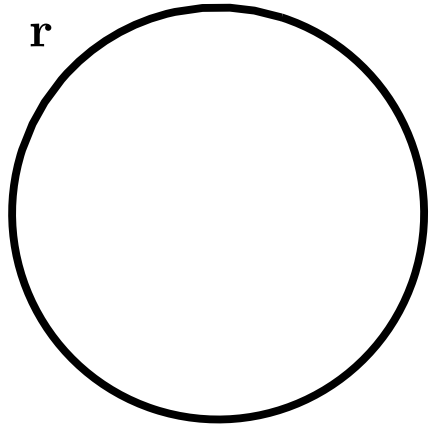

(a)
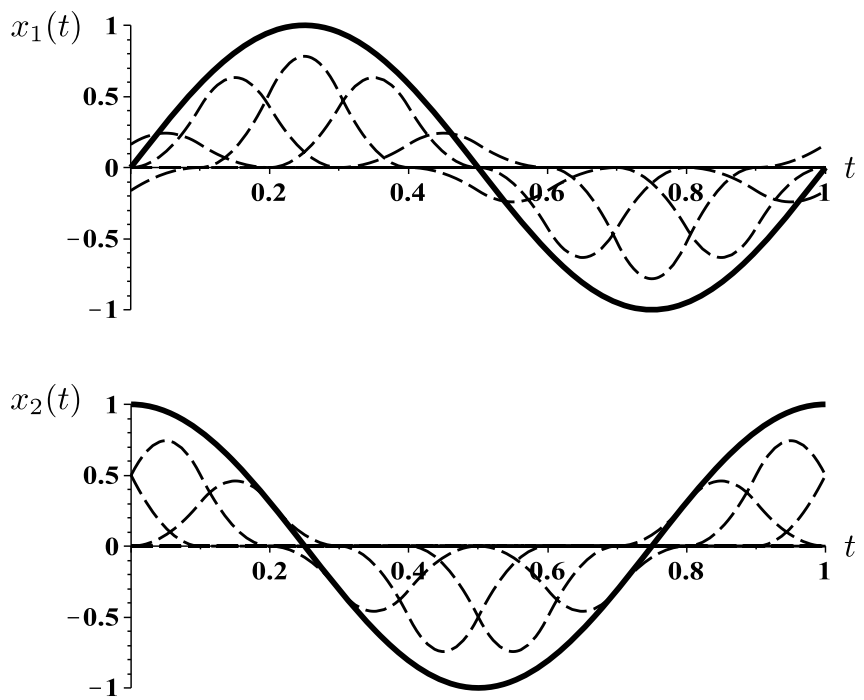

(b)

Fig. 2. Parametric representation of the unit circle (a) and its coordinate functions (b) with exponential B-splines and $M=10$. The dashed lines in (b) indicate the corresponding basis functions.

$$
\left\{\begin{array}{l}
\varsigma_{M}(t)=\frac{1}{4} \operatorname{sgn}(t) \frac{\sin ^{2}\left(\frac{\pi}{M} t\right)}{\sin ^{2} \frac{\pi}{M}}, \\
c_{M}^{S}=\left[1,1+2 \cos \frac{2 \pi}{M}, 1+2 \cos \frac{2 \pi}{M}, 1\right] .
\end{array}\right.
$$

Proof. Using (21), we see that $\varphi_{M}^{S}$ needs to be constructed from combinations of exponential B-splines with parameters $\boldsymbol{\alpha}=\left(0, \mathrm{j} \frac{2 \pi}{M},-\mathrm{j} \frac{2 \pi}{M}\right)$, which leaves $N=3$. Therefore, we have

$$
\varphi_{M}^{\mathrm{S}}(t)=\sum_{n=0}^{2} \lambda_{n}^{\mathrm{S}}[M] \frac{\mathrm{d}^{n}}{\mathrm{~d} t^{n}} \beta_{\alpha}(t-a) .
$$

This ensures that $\varphi_{M}^{\mathrm{S}}$ is the shortest generating function that reproduces constants and all sinusoids of unit period with $M$ coefficients. The constant-reproduction property is a direct consequence of using $\alpha_{1}=0$, and the sinusoid-reproduction property comes from applying Euler's identity to $\alpha_{2}=\mathrm{j} \frac{2 \pi}{M}$ and $\alpha_{3}=-\mathrm{j} \frac{2 \pi}{M}$.

In order to maximize the smoothness of the resulting generating function, the coefficients $\lambda_{1}^{S}[M]$ and $\lambda_{2}^{S}[M]$ in (30) must vanish. Since $\varphi_{M}^{S}$ reproduces constants, $\lambda_{0}^{S}[M]$ can be determined by imposing the partition-of-unity condition. From (7), we have that

$$
\lambda_{0}^{S}[M]=\left(\operatorname{sinc} \frac{1}{M}\right)^{-2} .
$$

An exponential B-spline parameterized by $\boldsymbol{\alpha}$ generates a Riesz basis if and only if $\left(\alpha_{m_{1}}-\alpha_{m_{2}}\right) \notin 2 \pi \mathrm{j} \mathbb{Z}$ for all purely imaginary pairs such that $m_{1} \neq m_{2}$. In our case, it is important to realize that this condition is satisfied if and only if $M \geqslant M_{0}=3$. In other words, at least three control points are needed to define our parametric curve.

Finally, a closed form for $\varphi_{M}^{S}$ is obtained by computing the inverse Fourier transform of

$$
\hat{\varphi}_{M}^{\mathrm{S}}(\omega)=\lambda_{0}^{\mathrm{S}}[M] \mathrm{e}^{\mathrm{j} \frac{3 \omega}{2}} \frac{1-\mathrm{e}^{-\mathrm{j} \omega}}{\mathrm{j} \omega} \frac{1-\mathrm{e}^{-\left(\mathrm{j} \omega-\mathrm{j} \frac{2 \pi}{M}\right)}}{\mathrm{j} \omega-\mathrm{j} \frac{2 \pi}{M}} \frac{1-\mathrm{e}^{-\left(\mathrm{j} \omega+\mathrm{j} \frac{2 \pi}{M}\right)}}{\mathrm{j} \omega+\mathrm{j} \frac{2 \pi}{M}},
$$

where we have set $a=-\frac{3}{2}$ in order to ensure that the basis function is centered.

We show in Fig. 3 some members of this family of functions for several values of $M$. We observe that they are continuous, with finite support of length $W=3$, and tend to be bump-like. Moreover, when $M \rightarrow \infty$, they converge to the quadratic B-spline. By a Maclaurin series expansion, we have that $\lim _{M \rightarrow \infty} \varsigma_{M}(t)=\frac{1}{4} \operatorname{sgn}(t) t^{2}$. Then, $\lim _{M \rightarrow \infty} C_{M}^{S}=[1,3,3,1]$ immediately implies that $\lim _{M \rightarrow \infty} \varphi_{M}^{S}=\beta^{2}$. This is because a polynomial B-spline of degree $n$ can be written as 


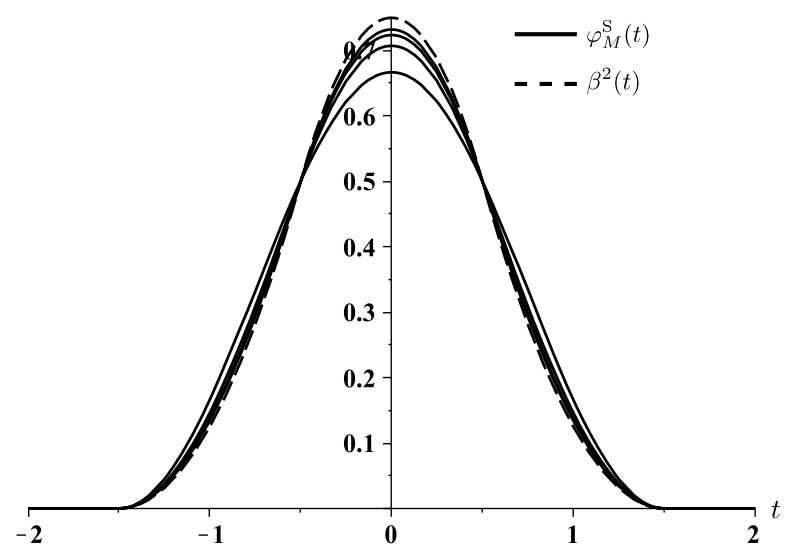

Fig. 3. Plot of a quadratic B-spline $\beta^{2}$ and of the generating functions in (29) for $M=3,4,5$, and 6 . The function with the lowest peak at $t=0$ corresponds to $M=3$, and, as $M$ increases, the central peak increases as well.

$$
\beta^{n}(t)=\sum_{k=0}^{n+1}(-1)^{k}\left(\begin{array}{c}
n+1 \\
k
\end{array}\right) \varsigma^{n}\left(t+\frac{n+1}{2}-k\right)
$$

where $\varsigma^{n}(t)=\frac{1}{2 n !} \operatorname{sgn}(t) t^{n}$. Note that the convergence of $\varphi_{M}^{S}$ to $\beta^{2}$ is point-wise. A piecewise expression of $\varphi_{M}^{S}$ can be obtained by expanding (29) into

$$
\varphi_{M}^{S}(t)=\frac{1}{1-\cos \frac{2 \pi}{M}} \begin{cases}\cos \frac{2 \pi|t|}{M} \cos \frac{\pi}{M}-\cos \frac{2 \pi}{M}, & 0 \leqslant|t|<\frac{1}{2}, \\ \left(\sin \frac{\pi(3 / 2-|t|)}{M}\right)^{2}, & \frac{1}{2} \leqslant|t|<\frac{3}{2}, \\ 0, & \frac{3}{2} \leqslant|t| .\end{cases}
$$

\subsubsection{Minimal-support interpolating basis for sinusoids}

As was suggested in Section 3.6, the generating function $\varphi_{M}$ can be tailored to satisfy the interpolating condition. We investigate now how this applies to the reproduction of ellipses and other trigonometry-related curves.

Corollary 2. The centered interpolating generating function with minimal support that satisfies all conditions in Section 2.3 and that reproduces sinusoids of unit period with $M$ coefficients is

$$
\varphi_{M}^{\mathrm{I}}(t)=\sum_{k=0}^{3}(-1)^{k} c_{M}^{\mathrm{S}}[k] \sec \frac{\pi}{M}\left(\varsigma_{M}\left(t+\frac{3}{2}-k\right)-\frac{1}{16}\left(\sec \frac{\pi}{2 M}\right)^{2} \operatorname{sgn}\left(t+\frac{3}{2}-k\right)\right) .
$$

Proof. Following the same approach as when constructing $\varphi_{M}^{S}$, we see that $\varphi_{M}^{\mathrm{I}}$ needs to be constructed from combinations of exponential B-splines with parameters $\alpha=\left(0, \mathrm{j} \frac{2 \pi}{M},-\mathrm{j} \frac{2 \pi}{M}\right)$. Therefore, we have that

$$
\varphi_{M}^{\mathrm{I}}(t)=\sum_{n=0}^{2} \lambda_{n}^{\mathrm{I}}[M] \frac{\mathrm{d}^{n}}{\mathrm{~d} t^{n}} \beta_{\boldsymbol{\alpha}}(t-a) .
$$

In order to fulfill the interpolating condition, $\lambda_{0}^{\mathrm{I}}[M], \lambda_{1}^{\mathrm{I}}[M]$, and $\lambda_{2}^{\mathrm{I}}[M]$ in (30) must satisfy a linear system of equations. If we set $a=-\frac{3}{2}$ in order to ensure that the basis function is centered, we end up with

$$
\begin{aligned}
& \lambda_{0}^{\mathrm{I}}[M]=1, \\
& \lambda_{1}^{\mathrm{I}}[M]=0, \\
& \lambda_{2}^{\mathrm{I}}[M]=\left(\frac{M}{2 \pi}\right)^{2}\left(1-\sec \frac{\pi}{M}\right) .
\end{aligned}
$$

In this case, the interpolating $\varphi_{M}^{\mathrm{I}}$ is a Riesz basis if and only if $M \geqslant 3$, a condition that we already encountered in the case of Corollary 1. Finally, a closed form for $\varphi_{M}^{\mathrm{I}}$ is obtained by applying an inverse Fourier transform to 


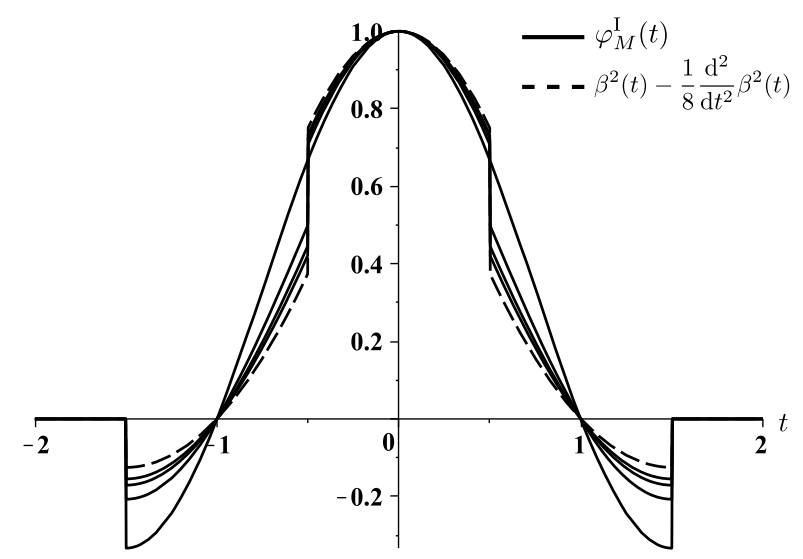

Fig. 4. Plot of the third-order I-MOMS $\left(\beta^{2}-\frac{1}{8} \ddot{\beta}\right)$ and of the generating functions in (32) for $M=3,4,5$, and 6 . Among the different $\varphi_{M}^{\mathrm{I}}$, the function with the least pronounced discontinuity at $t= \pm \frac{3}{2}$ corresponds to $M=3$, and, as $M$ increases, the jump of the discontinuity increases as well. For $M=3$, $\varphi_{M}^{\mathrm{I}}$ is continuous at $t= \pm \frac{1}{2}$, but discontinuous at $t= \pm \frac{3}{2}$.

$$
\begin{aligned}
\hat{\varphi}_{M}^{\mathrm{I}}(\omega)= & \lambda_{0}^{\mathrm{I}}[M] \mathrm{e}^{\mathrm{j} \frac{3 \omega}{2}} \frac{1-\mathrm{e}^{-\mathrm{j} \omega}}{\mathrm{j} \omega} \frac{1-\mathrm{e}^{-\left(\mathrm{j} \omega-\mathrm{j} \frac{2 \pi}{M}\right)}}{\mathrm{j} \omega-\mathrm{j} \frac{2 \pi}{M}} \frac{1-\mathrm{e}^{-\left(\mathrm{j} \omega+\mathrm{j} \frac{2 \pi}{M}\right)}}{\mathrm{j} \omega+\mathrm{j} \frac{2 \pi}{M}} \\
& +\lambda_{2}^{\mathrm{I}}[M](\mathrm{j} \omega)^{2} \mathrm{e}^{\mathrm{j} \frac{3 \omega}{2}} \frac{1-\mathrm{e}^{-\mathrm{j} \omega}}{\mathrm{j} \omega} \frac{1-\mathrm{e}^{-\left(\mathrm{j} \omega-\mathrm{j} \frac{2 \pi}{M}\right)}}{\mathrm{j} \omega-\mathrm{j} \frac{2 \pi}{M}} \frac{1-\mathrm{e}^{-\left(\mathrm{j} \omega+\mathrm{j} \frac{2 \pi}{M}\right)}}{\mathrm{j} \omega+\mathrm{j} \frac{2 \pi}{M}} .
\end{aligned}
$$

We show in Fig. 4 some members of this family of functions for several values of $M$. We observe that they share a finite support of length $W=3$. As we increase $M, \varphi_{M}^{\mathrm{I}}$ converges to $\beta^{2}-\frac{1}{8} \ddot{\beta}^{2}$, which is the third-order I-MOMS described in Blu et al. (2001). A piecewise expression of $\varphi_{M}^{\mathrm{I}}$ can be obtained by expanding (31) into

$$
\varphi_{M}^{\mathrm{I}}(t)= \begin{cases}\frac{\cos \frac{2 \pi t}{M}-\cos \frac{2 \pi}{M}}{1-\cos \frac{2 \pi}{M}}, & 0 \leqslant|t|<\frac{1}{2}, \\ \frac{4 \cos ^{2} \frac{\pi}{M}+4 \cos \frac{\pi}{M}+1}{8 \cos \frac{\pi}{M}\left(\cos \frac{\pi}{M}+1\right)}, & |t|=\frac{1}{2}, \\ \frac{\cos \frac{\pi}{M}-\cos \frac{2 \pi(3 / 2-|t|)}{M}}{2\left(1-\cos \frac{2 \pi}{M}\right) \cos \frac{\pi}{M}}, & \frac{1}{2} \leqslant|t|<\frac{3}{2}, \\ \frac{-1}{8 \cos \frac{\pi}{M}\left(\cos \frac{\pi}{M}+1\right)}, & |t|=\frac{3}{2}, \\ 0, & |t|>\frac{3}{2} .\end{cases}
$$

\subsubsection{Refinability of the proposed bases}

As discussed in Section 4.2, not all members of the family of functions given by Theorem 2 are refinable. Here, we show the multiresolution properties of the proposed basis functions that reproduce sinusoids.

When imposing maximal smoothness, it is straightforward to verify that the basis function $\varphi_{M}^{S}$ is refinable since it is proportional to a refinable exponential B-spline. To build the associated refinement mask, we have to take into account that $a$ is a half integer. Therefore, there is a half-integer shift in the parameterization every time we apply the refinement. That means that a curve $\mathbf{r}_{M}$ built with $M$ coefficients and the same curve expressed with $2 M$ coefficients satisfy

$$
\mathbf{r}_{M}(t)=\lambda_{0}^{S}[M] \sum_{k=-\infty}^{\infty} \mathbf{c}_{M}[k] \beta_{\boldsymbol{\alpha}}\left(M t-k+\frac{3}{2}\right)=\lambda_{0}^{S}[2 M] \sum_{k=-\infty}^{\infty} \mathbf{c}_{2 M}[k] \beta_{\frac{\alpha}{2}}\left(2 M t-k+\frac{3}{2}-\frac{1}{2}\right) .
$$

The dependency between the two sequences of coefficients can be stated as

$$
\mathbf{c}_{2 M}[k]=\frac{\lambda_{0}^{S}[M]}{\lambda_{0}^{S}[2 M]} \sum_{l=-\infty}^{\infty} \mathbf{c}_{M}[l] h_{\frac{\alpha}{2}}[k+2-2 l]=\left(\frac{\operatorname{sinc} \frac{1}{2 M}}{\operatorname{sinc} \frac{1}{M}}\right)^{2}\left(\left(\mathbf{c}_{M}\right)_{\uparrow 2} * h_{\frac{\alpha}{2}}\right)[k+2],
$$

where $\left(\mathbf{c}_{M}\right)_{\uparrow 2}$ is the $\mathbf{c}_{M}$ sequence upsampled by a factor of 2 . It is interesting to note that the filter $h_{\frac{\alpha}{2}}$ is equal to the sequence $c_{M}^{S}$ in the expression of $\varphi_{M}^{S}$ in (29). We identify the refinement filter $\tilde{h}_{\frac{\alpha}{2}}$ described in Section 4.2 as a shifted and scaled version of the refinement filter $h_{\frac{\alpha}{2}}$ of the exponential B-spline $\beta_{\alpha}$. 
When imposing the interpolation property, it can be shown that, for the particular choice $\lambda_{0}^{\mathrm{I}}[M], \lambda_{1}^{\mathrm{I}}[M]$, and $\lambda_{2}^{\mathrm{I}}[M]$ leads to a ratio $\frac{\Lambda_{M}(\mathrm{j} 2 \omega)}{\Lambda_{2 M}(\mathrm{j} \omega)}$ that is not $2 \pi$-periodic. Thus, the multiresolution spaces are not nested, and no refinement mask exists. Meanwhile, $\varphi_{M}^{\mathrm{I}}$ is unique due to the restrictions introduced by the interpolatory condition, and there is no remaining degree of freedom to be used to increase the regularity or to improve the multiresolution properties of the basis function.

\subsubsection{Additional refinable bases}

In this section, we illustrate a constructive procedure to design new refinable schemes. In particular, we focus on the particular case where the ratio $\frac{\Lambda_{M}(\mathrm{j} 2 \omega)}{\Lambda_{2 M}(\mathrm{j} \omega)}$ is constant. This can be achieved by imposing scaling conditions over the $N^{\prime}$ roots $\left\{\gamma_{n}\right\}_{n=1 \ldots N^{\prime}}$ of the polynomial $\Lambda_{M}(\mathrm{j} \omega)$. Then, we have that

$$
\Lambda(\mathrm{j} \omega)=\lambda_{N^{\prime}}[M] \prod_{n=1}^{N^{\prime}}\left(\mathrm{j} \omega-\gamma_{n}[M]\right),
$$

where $N^{\prime}<N$ and where we have made explicit the dependence of the roots with respect to $M$. Note that there is a one-toone dependence between the elements of the set $\left\{\lambda_{n}\right\}_{n=0 \ldots N-1}$ and the roots of the polynomial $\left\{\gamma_{n}\right\}_{n=1 \ldots N^{\prime}}$, up to a scaling factor. In particular, if we choose the roots such that

$$
\gamma_{n}[2 M]=\frac{\gamma_{n}[M]}{2}
$$

for all $n$, then the quantity

$$
\begin{array}{rlrl}
\frac{\Lambda_{M}(\mathrm{j} 2 \omega)}{\Lambda_{2 M}(\mathrm{j} \omega)} & =\frac{\lambda_{N^{\prime}}[M] \prod_{n=1}^{N^{\prime}}\left(\mathrm{j} 2 \omega-\gamma_{n}[M]\right)}{\lambda_{N^{\prime}}[2 M] \prod_{n=1}^{N^{\prime}}\left(\mathrm{j} \omega-\gamma_{n}[2 M]\right)} & & \text { by definition } \\
& =\frac{\lambda_{N^{\prime}}[M] 2^{N^{\prime}} \prod_{n=1}^{N^{\prime}}\left(\mathrm{j} \omega-\gamma_{n}[M] / 2\right)}{\lambda_{N^{\prime}}[2 M] \prod_{n=1}^{N^{\prime}}\left(\mathrm{j} \omega-\gamma_{n}[2 M]\right)} & \text { factoring } \\
& =2^{N^{\prime}} \frac{\lambda_{N^{\prime}}[M]}{\lambda_{N^{\prime}}[2 M]} & \text { by (33) }
\end{array}
$$

is independent of $\omega$ and the resulting function $\varphi$ is refinable. This particular multiresolution scheme where the roots of $\Lambda_{M}(\mathrm{j} \omega)$ satisfy (33) is intimately related to the generalized exponential B-splines proposed in Khalidov and Unser (2006).

To build new refinable basis functions that reproduce sinusoids, we can choose the roots $\left\{\gamma_{n}^{\mathrm{R}}\right\}_{n=1 \ldots N^{\prime}}$ of $\Lambda_{M}(\mathrm{j} \omega)$ such that $\gamma_{n}^{\mathrm{R}}[2 M]=\gamma_{n}^{\mathrm{R}}[M] / 2$. The number of roots $N^{\prime}$ determines which is the maximum non-zero element in the sequence $\left\{\lambda_{n}^{\mathrm{R}}\right\}_{n=0 . .2}$, and therefore the smoothness of the resulting basis function.

The particular choice of $\gamma_{1}^{\mathrm{R}}[M]=-\gamma_{2}^{\mathrm{R}}[M]=\frac{1}{M}$ and $a=-\frac{3}{2}$ defines a refinable, centered, and symmetric generating function with minimal support that reproduces sinusoids of unit period with $M$ coefficients. These roots determine the set of parameters $\left\{\lambda_{n}^{R}\right\}_{n=0 . .2}$ up to a scaling constant as

$$
\begin{aligned}
& \lambda_{0}^{\mathrm{R}}[M]=-\frac{\lambda_{2}^{\mathrm{R}}[M]}{M^{2}}, \\
& \lambda_{1}^{\mathrm{R}}[M]=0, \\
& \lambda_{2}^{\mathrm{R}}[M]=\lambda_{2}^{\mathrm{R}}[M] .
\end{aligned}
$$

Then, the resulting generating function is

$$
\varphi_{M}^{\mathrm{R}}(t)=-\frac{\lambda_{2}^{\mathrm{R}}[M]}{M^{2}} \beta_{\alpha}\left(M t-k+\frac{3}{2}\right)+\lambda_{2}^{\mathrm{R}}[M] \ddot{\beta}_{\alpha}\left(M t-k+\frac{3}{2}\right) .
$$

We show in Fig. 5 some members of this family of functions for several values of $M$. We choose $\lambda_{2}^{\mathrm{R}}[M]$ such that the $L_{2}$ norm of $\varphi_{M}^{\mathrm{R}}(t)$ is unitary. We observe that they share a finite support of length $W=3$.

Our choice of $\left\{\gamma_{n}^{\mathrm{R}}\right\}_{n=1 \ldots N^{\prime}}$ is arbitrary and corresponds to one particular case where the resulting generating function is symmetric and non-smooth. Other choices would lead to asymmetric functions and other degrees of smoothness.

\subsubsection{Order of approximation}

The notion of order of approximation is crucial in approximation theory since it governs the rate of decrease of the approximation error as the sampling step vanishes. Specifically, in the periodic stationary case, the approximation order

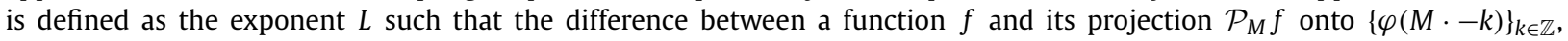
or equivalently in $\left\{\varphi_{\text {per }}(M \cdot-k)\right\}_{k=[0 \ldots M-1]}$, tends to zero. In direct analogy with the classical Strang-and-Fix theory of approximation for the non-periodic case, it has been shown in Jacob et al. (2002) that the error for the periodic case can be bounded by 


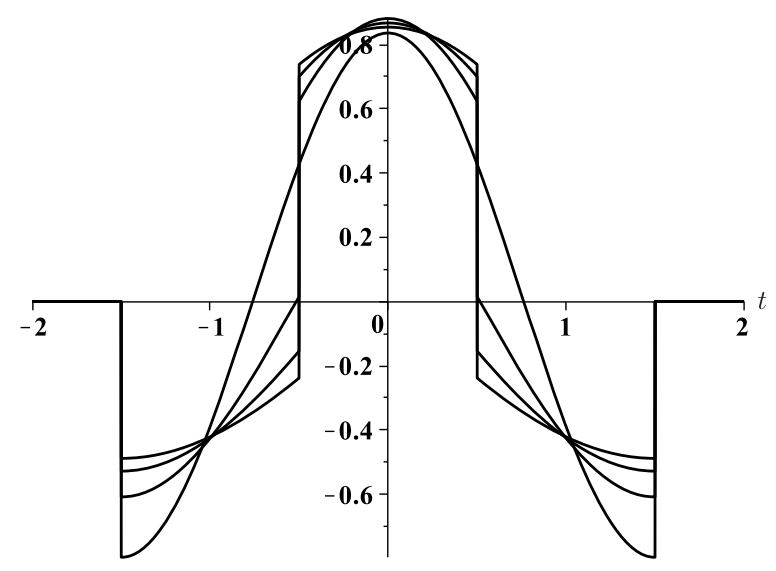

Fig. 5. Plot of the generating functions in (34) for $M=3,4,5$, and 6 . Among the different $\varphi_{M}^{\mathrm{R}}$, the function with the most pronounced discontinuity at $t= \pm \frac{3}{2}$ corresponds to $M=3$, and, as $M$ increases, the jump of the discontinuity decreases.

Table 1

Summary of the properties of $\varphi_{M}^{S}$ and $\varphi_{M}^{I}$.

\begin{tabular}{lll}
\hline & $\varphi_{M}^{\mathrm{S}}$ & $\varphi_{M}^{\mathrm{I}}$ \\
\hline Parameters & $\lambda_{0}^{\mathrm{S}}[M]=\left(\operatorname{sinc} \frac{1}{M}\right)^{-2}$ & $\lambda_{0}^{\mathrm{I}}[M]=1$ \\
& $\lambda_{1}^{\mathrm{S}}[M]=0$ & $\lambda_{1}^{\mathrm{I}}[M]=0$ \\
Smoothness & $\lambda_{2}^{\mathrm{S}}[M]=0$ & $\lambda_{2}^{\mathrm{I}}[M]=\left(\frac{M}{2 \pi}\right)^{2}\left(1-\sec \frac{\pi}{M}\right)$ \\
Order of approximation & Discontinuous & $\mathcal{C}^{1}(\mathbb{R})$ \\
Limit $(M \rightarrow \infty)$ & $\mathcal{O}\left(M^{-3}\right)$ & $\mathcal{O}\left(M^{-3}\right)$ \\
Refinable & $\beta^{2}$ & $\beta^{2}-\frac{1}{8} \ddot{\beta}^{2}$ \\
\hline
\end{tabular}

$$
\left\|f-\mathcal{P}_{M} f\right\|_{L_{2}([0,1])} \leqslant C_{\varphi} M^{-L}\|f\|_{L_{2}([0,1])},
$$

where $C_{\varphi}$ is a constant that only depends on the particular choice of $\varphi$. An analogous result for the nonstationary case can be obtained using the concept of asymptotically equivalent subdivision schemes presented in Dyn and Levin (1995). We say that $\varphi_{M}$ and $\tilde{\varphi}$ define equivalent multiresolution schemes of order $\gamma$ if and only if

$$
\left\|\tilde{\mathcal{P}}_{M} f-\mathcal{P}_{M} f\right\|_{L_{2}([0,1])}=\mathcal{O}\left(M^{-\gamma}\right),
$$

where $\mathcal{P}_{M} f$ denotes the projection of $f$ onto $\left\{\varphi_{M}(N \cdot-k)\right\}_{k \in \mathbb{Z}}$ with $N=M$, and $\tilde{\mathcal{P}}_{M} f$ denotes the projection of $f$ onto

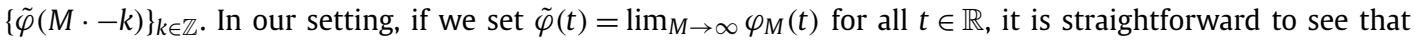

$$
\left\|f-\mathcal{P}_{M} f\right\|_{L_{2}([0,1])} \leqslant\left\|f-\tilde{\mathcal{P}}_{M} f\right\|_{L_{2}([0,1])}+\left\|\tilde{\mathcal{P}}_{M} f-\mathcal{P}_{M} f\right\|_{L_{2}([0,1])}=\mathcal{O}\left(M^{-\min (L, \gamma)}\right) .
$$

Therefore, if the $\varphi_{M}$ and $\tilde{\varphi}$ define multiresolution schemes of order high enough, the rate of decay of the error is the same for the nonstationary and the stationary case.

By taking the limit $M \rightarrow \infty$ on $\varphi_{M}^{\mathrm{S}}$ and $\varphi_{M}^{\mathrm{I}}$, we can observe that such functions converge to the classical quadratic B-spline $\beta^{2}$ and to the third-order I-MOMS $\beta^{2}-\frac{1}{8} \ddot{\beta}^{2}$ derived in Blu et al. (2001), respectively. Both generating functions are known to have the same order of approximation $L=3$. The main difference between them lies in the constant that multiplies the $M^{-3}$ factor. This factor is more favorable in the case of the quadratic B-spline than in the case of the thirdorder I-MOMS. Thus, in general, the approximation offered by the quadratic B-spline is more accurate than the one offered by the I-MOMS. This property carries over to $\varphi_{M}^{\mathrm{S}}$ and $\varphi_{M}^{\mathrm{I}}$ when $M \rightarrow \infty$.

\subsection{Reproduction of higher-order harmonics}

We now present a constructive procedure to extend the ellipse-reproduction properties of our curves to higher-order harmonics. This problem was already approached using Fourier descriptors (Zahn and Roskies, 1972). Since our basis functions are capable of perfectly reproducing sinusoids, the classical family of Fourier descriptors becomes a special class of our construction. It must be noted, though, that our bases have a finite support, a property which is lacking in Fourier descriptors.

We say that a parametric curve defined by $M$ vectorial coefficients and by a generating function $\varphi_{M}$ reproduces higherorder harmonics up to order $L$ if there exist two $M$-periodic sequences $\left\{c_{l, c}[k]\right\}_{k \in \mathbb{Z}}$ and $\left\{c_{l, s}[k]\right\}_{k \in \mathbb{Z}}$ for every $1 \leqslant l \leqslant L$ such that 


$$
\begin{aligned}
& \cos (2 \pi l t)=\sum_{k=0}^{M-1} c_{l, c}[k] \varphi_{M, \operatorname{per}}(M t-k), \\
& \sin (2 \pi l t)=\sum_{k=0}^{M-1} c_{l, s}[k] \varphi_{M, p e r}(M t-k) .
\end{aligned}
$$

Such a curve is able to reproduce all modes up to order $L$ for each component. Like in the case of the sinusoids, it is possible to represent any initial phase using linear combinations of the two sequences of coefficients in (36) and (37). We recall that, using Euler's identity and the multinomial theorem, related functions such as $\left(\cos (2 \pi \cdot)^{l}\right.$ and $(\sin (2 \pi \cdot))^{l}$, with $1 \leqslant l \leqslant L$, can also be expressed as linear combinations of elements from $\{\cos (2 \pi l \cdot), \sin (2 \pi l \cdot)\}_{1 \leqslant l \leqslant L}$. This ensures that the functions $(\cos (2 \pi \cdot))^{l}$ and $(\sin (2 \pi \cdot))^{l}$ are expressible with the same basis functions $\varphi_{M}$ or $\varphi_{M \text {,per }}$.

5.2.1. Minimal-support basis of maximum smoothness for higher-order harmonics

Corollary 3. The centered generating function with minimal support and maximal smoothness that satisfies all conditions in Section 2.3 and that reproduces higher-order harmonics up to order $L$ with $M$ coefficients is

$$
\varphi_{M}^{S}(t)=\lambda_{0}[M] \beta_{\alpha}\left(t+\frac{2 L+1}{2}\right),
$$

where $\boldsymbol{\alpha}$ contains only $\{0\},\left\{\mathrm{j} \frac{2 \pi}{M} k\right\}_{k \in[1 \ldots L]}$, and $\left\{-\mathrm{j} \frac{2 \pi}{M} k\right\}_{k \in[1 \ldots L]}$, and where $\lambda_{0}[M]$ is an appropriate normalizing constant.

Proof. The proof follows the same strategy as in Corollary 1. The choice of the collection $\alpha$ and the size of the support $N=2 L+1$ is given by Theorem 2 . The parameters $\lambda_{1}[M], \ldots, \lambda_{L}[M]$ are set to zero to maximize the smoothness of $\varphi_{M}^{S}$, and $\lambda_{0}[M]$ is fixed in such a way that $\varphi_{M}^{\mathrm{S}}$ satisfies the partition-of-unity condition, which yields

$$
\lambda_{0}[M]=\frac{1}{\sum_{k=1}^{2 L-1} \beta_{\boldsymbol{\alpha}}\left(k+\frac{1}{2}\right)} .
$$

We recall that exponential B-splines parameterized by $\alpha$ form a Riesz basis if and only if $\left(\alpha_{m_{1}}-\alpha_{m_{2}}\right) \notin 2 \pi \mathrm{j} \mathbb{Z}$ for all pairs such that $m_{1} \neq m_{2}$. In our case, this condition is satisfied if $M \geqslant 2 L+1$. Finally, the shift parameter is set to $a=-\frac{2 L+1}{2}$ to ensure that the generating function is centered.

It should be noted that the smoothest basis function corresponds to a normalized trigonometric spline, which was defined as a piecewise trigonometric function by Schoenberg (1964).

\subsubsection{Parametric expansion of higher-order harmonics}

Here, we determine the sequence of $M$ vector coefficients that reproduce the higher-order harmonics using the generating function $\varphi_{M}^{S}$ given in (38). We start by recalling the exponential-reproducing property of the exponential B-splines

$$
\mathrm{e}^{\alpha t}=\sum_{k=-\infty}^{\infty} \mathrm{e}^{\alpha k} \beta_{(\alpha)}(t-k) .
$$

Setting $\alpha=\mathrm{j} \frac{2 \pi l}{M}$ with $1 \leqslant l \leqslant L$, we see that $\beta_{\left(\mathrm{j} \frac{2 \pi l}{M}\right)}$ reproduces the complex exponential $\mathrm{e}^{\mathrm{j} \frac{2 \pi l}{M} t}$. If we now convolve both sides of (39) with $\beta_{\boldsymbol{\alpha} \backslash \mathrm{j} \frac{2 \pi l}{M} \text { ) }}$, we get that

$$
\left(\beta_{\left.\boldsymbol{\alpha} \backslash \mathrm{j} \frac{2 \pi l}{M}\right)} * \mathrm{e}^{\mathrm{j} \frac{2 \pi l}{M}}\right)(t)=\sum_{k=-\infty}^{\infty} \mathrm{e}^{\mathrm{j} \frac{2 \pi l}{M} k} \underbrace{\left(\beta_{\left(\mathrm{j} \frac{2 \pi l}{M}\right)} * \beta_{\left.\boldsymbol{\alpha} \backslash \mathrm{j} \frac{2 \pi l}{M}\right)}\right)(t-k)}_{\frac{1}{\lambda_{0}[M]} \varphi_{M}^{S}\left(t-\frac{2 L+1}{2}-k\right)},
$$

where we have used the definition of $\varphi_{M}^{S}$ from (38), along with the fact that the convolution operator commutes with the shift operator. To simplify the left-hand side, we invoke an important property of linear shift-invariant (LSI) systems: complex exponentials are eigenfunctions of LSI operators. By virtue of this property, if the complex exponential $\mathrm{e}^{\mathrm{j} \alpha t}$ is presented at the input of a system specified by the impulse response $h$, then its output is given by $\hat{h}(\alpha) \mathrm{e}^{\mathrm{j} \alpha t}$, where $\hat{h}$ denotes the Fourier transform of $h$. If we consider $\beta_{\boldsymbol{\alpha} \backslash\left(\mathrm{j} \frac{2 \pi l}{M}\right)}$ as the impulse response of an LSI system, then

$$
\left(\beta_{\boldsymbol{\alpha} \backslash\left(\mathrm{j} \frac{2 \pi l}{M}\right)} * \mathrm{e}^{\mathrm{j} \frac{2 \pi l}{M} \cdot}\right)(t)=\left.\hat{\beta}_{\boldsymbol{\alpha} \backslash\left(\mathrm{j} \frac{2 \pi l}{M}\right)}(\omega)\right|_{\omega=\frac{2 \pi l}{M}} \mathrm{e}^{\mathrm{j} \frac{2 \pi l}{M} t} .
$$

Therefore, we have that 


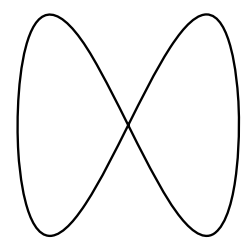

(a)

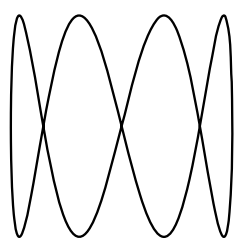

(b)

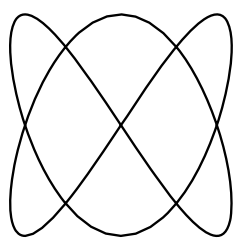

(c)

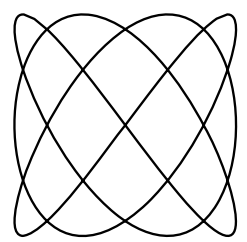

(d)

Fig. 6. Lissajous curves.

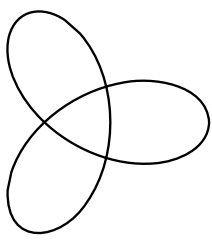

(a)

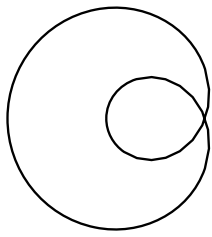

(a)

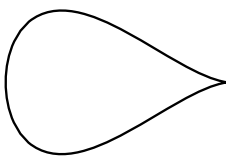

(a)

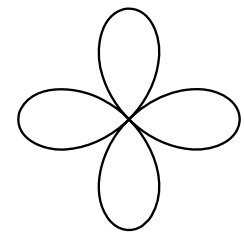

(b)

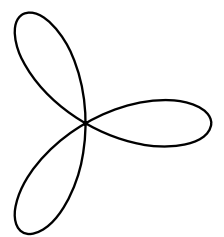

(c)

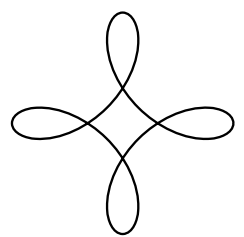

(d)

Fig. 7. Hypotrochoid curves.

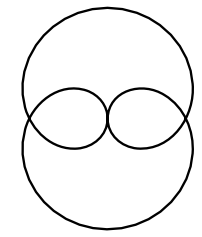

(b)

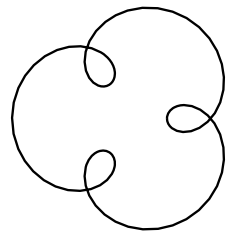

(c)

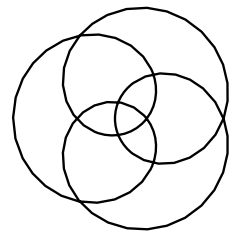

(d)

Fig. 8. Epitrochoid curves.

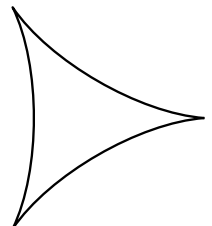

(b)

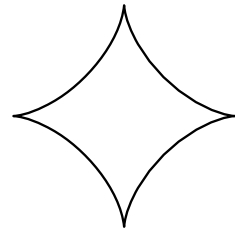

(c)

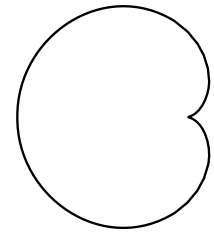

(d)

Fig. 9. Other curves: (a) Teardrop, (b) Deltoid, (c) Astroid, (d) Cardioid.

$$
\mathrm{e}^{\mathrm{j} \frac{2 \pi l}{M} t}=\sum_{k=-\infty}^{\infty} \mathrm{e}^{\mathrm{j} \frac{2 \pi l}{M} k} \frac{1}{\left.\lambda_{0}[M] \hat{\beta}_{\left.\boldsymbol{\alpha} \backslash \mathrm{j} \frac{2 \pi l}{M}\right)}(\omega)\right|_{\omega=\frac{2 \pi l}{M}}} \varphi_{M}^{\mathrm{S}}\left(t-\frac{2 L+1}{2}-k\right) .
$$

By flipping the sign of $\alpha$, we can easily obtain an analogous result for the reproduction of $\mathrm{e}^{-\mathrm{j} \frac{2 \pi l}{M} t}$. Finally, by using both results, we have that

$$
\begin{aligned}
& \cos \left(2 \pi l\left(t+\frac{2 L+1}{2 M}\right)\right)=\sum_{k=-\infty}^{\infty} c_{1}[k] \varphi_{M}^{S}(M t-k), \\
& \sin \left(2 \pi l\left(t+\frac{2 L+1}{2 M}\right)\right)=\sum_{k=-\infty}^{\infty} c_{2}[k] \varphi_{M}^{S}(M t-k),
\end{aligned}
$$

where

$$
\begin{aligned}
& c_{1}[k]=\frac{1}{2 \lambda_{0}[M]}\left(\frac{\mathrm{e}^{\mathrm{j} \frac{2 \pi l}{M} k}}{\left.\hat{\beta}_{\boldsymbol{\alpha} \backslash\left(\mathrm{j} \frac{2 \pi l}{M}\right)}(\omega)\right|_{\omega=\frac{2 \pi l}{M}}}+\frac{\mathrm{e}^{-\mathrm{j} \frac{2 \pi l}{M} k}}{\left.\hat{\beta}_{\boldsymbol{\alpha} \backslash\left(-\mathrm{j} \frac{2 \pi l}{M}\right)}(\omega)\right|_{\omega=-\frac{2 \pi l}{M}}}\right), \\
& c_{2}[k]=\frac{1}{2 \mathrm{j} \lambda_{0}[M]}\left(\frac{\mathrm{e}^{\mathrm{j} \frac{2 \pi l}{M} k}}{\left.\hat{\beta}_{\left.\boldsymbol{\alpha} \backslash \mathrm{j} \frac{2 \pi l}{M}\right)}(\omega)\right|_{\omega=\frac{2 \pi l}{M}}}-\frac{\mathrm{e}^{-\mathrm{j} \frac{2 \pi l}{M} k}}{\left.\hat{\beta}_{\boldsymbol{\alpha} \backslash\left(-\mathrm{j} \frac{2 \pi l}{M}\right)}(\omega)\right|_{\omega=-\frac{2 \pi l}{M}}}\right) .
\end{aligned}
$$


Table 2

Coefficients for the curves shown in Figs. 6, 7, 8, and 9.

\begin{tabular}{lll}
\hline Curve & $c_{x_{1}}$ & $c_{x_{2}}$ \\
\hline Lissajous (a) & $c_{1, \mathrm{~s}}$ & $c_{2, \mathrm{~s}}$ \\
Lissajous (b) & $c_{1, \mathrm{~s}}$ & $c_{4, \mathrm{~s}}$ \\
Lissajous (c) & $c_{2, \mathrm{~s}}$ & $c_{3, \mathrm{~s}}$ \\
Lissajous (d) & $c_{3, \mathrm{~s}}$ & $c_{4, \mathrm{~s}}$ \\
Hypotrochoid (a) & $2 c_{1, \mathrm{c}}+3 c_{2, \mathrm{c}}$ & $2 c_{1, \mathrm{~s}}-3 c_{2, \mathrm{~s}}$ \\
Hypotrochoid (b) & $c_{1, \mathrm{c}}+c_{3, \mathrm{c}}$ & $c_{1, \mathrm{~s}}-c_{3, \mathrm{~s}}$ \\
Hypotrochoid (c) & $c_{1, \mathrm{c}}+c_{2, \mathrm{c}}$ & $c_{1, \mathrm{~s}}-c_{2, \mathrm{~s}}$ \\
Hypotrochoid (d) & $3 c_{1, \mathrm{c}}+2 c_{3, \mathrm{c}}$ & $3 c_{1, \mathrm{~s}}-2 c_{3, \mathrm{~s}}$ \\
Epitrochoid (a) & $2 c_{1, \mathrm{c}}-3 c_{2, \mathrm{c}}$ & $2 c_{1, \mathrm{~s}}-3 c_{2, \mathrm{~s}}$ \\
Epitrochoid (b) & $c_{1, \mathrm{c}}-c_{3, \mathrm{c}}$ & $c_{1, \mathrm{~s}}-c_{3, \mathrm{~s}}$ \\
Epitrochoid (c) & $2 c_{1, \mathrm{c}}-c_{4, \mathrm{c}}$ & $2 c_{1, \mathrm{~s}}-c_{4, \mathrm{~s}}$ \\
Epitrochoid (d) & $4 c_{1, \mathrm{c}}-5 c_{4, \mathrm{c}}$ & $4 c_{1, \mathrm{~s}}-5 c_{4, \mathrm{~s}}$ \\
Teardrop & $4 c_{1, \mathrm{c}}$ & $2 c_{1, \mathrm{~s}}-c_{2, \mathrm{~s}}$ \\
Deltoid & $2 c_{1, \mathrm{c}}+c_{2, \mathrm{c}}$ & $2 c_{1, \mathrm{~s}}-c_{2, \mathrm{~s}}$ \\
Astroid & $3 c_{1, \mathrm{c}}+c_{3, \mathrm{c}}$ & $3 c_{1, \mathrm{~s}}-c_{3, \mathrm{~s}}$ \\
Cardioid & $2 c_{1, \mathrm{c}}-c_{2, \mathrm{c}}$ & $2 c_{1, \mathrm{~s}}-c_{2, \mathrm{~s}}$ \\
\hline
\end{tabular}

Note that the sequences $c_{1}$ and $c_{2}$ can be considered $M$-periodic and that the summations in (40) and (41) can be reduced to finite ones if we make use of the periodized basis functions given in (4). We have expressed in (40) and (41) how to compute the vector coefficients for reproducing sinusoids and initial phase. The appropriate linear combination of $c_{1}$ and $c_{2}$ allows one to change arbitrarily the initial phase.

In order to illustrate the reproduction capabilities of the proposed model, we designed a basis function capable of reproducing some classical harmonic curves (Von Seggern, 1993). In particular, we tailored $\varphi_{M}^{S}$ in (38) with $L=4$ and $M=9$, which lead to $\alpha=\left(0, \mathrm{j} \frac{2 \pi}{9},-\mathrm{j} \frac{2 \pi}{9}, \ldots, \mathrm{j} \frac{8 \pi}{9},-\mathrm{j} \frac{8 \pi}{9}\right)$. We show some members of the Lissajous, Hypotrochoid, and Epitrochoid families in Figs. 6, 7, and 8, respectively. More singular examples like the Teardrop, the Deltoid, the Astroid, and the Cardioid are shown in Fig. 9. The coefficients for each coordinate function can be found in Table 2.

\section{Conclusions}

We have proposed a new family of basis functions that we use to represent planar curves. We were able to single out the basis of shortest support that allows one to reproduce exponential polynomials. Under the appropriate circumstances, these basis functions may form a natural multiscale hierarchy. In these cases, we specified multiresolution algorithms and subdivision schemes for the representation of geometric closed curves. We were able to characterize the order of approximation of such nonstationary multiresolution schemes. We exemplified our method by constructing minimal-support bases that reproduce ellipses and higher-order harmonics. In particular we tailored these bases to obtain maximal-smoothness basis functions, and interpolatory basis functions. We took advantage of the theoretical developments of this paper to build efficient active contours, which we present in the companion paper (Delgado-Gonzalo et al., 2011).

\section{References}

Aldroubi, A., Unser, M., 1994. Sampling procedures in function spaces and asymptotic equivalence with Shannon's sampling theory. Numerical Functional Analysis and Optimization 15, 1-21.

Beccari, C., Casciola, G., Romani, L., 2007. A non-stationary uniform tension controlled interpolating 4-point scheme reproducing conies. Computer Aided Geometric Design 24, 1-9.

Beccari, C., Casciola, G., Romani, L., 2009. Shape controlled interpolatory ternary subdivision. Applied Mathematics and Computation 215, 916-927.

Blu, T., Unser, M., 1999. Quantitative Fourier analysis of approximation techniques: Part I-Interpolators and projectors. IEEE Transactions on Signal Processing 47, 2783-2795.

Blu, T., Thévenaz, P., Unser, M., 2001. MOMS: Maximal-order interpolation of minimal support. IEEE Transactions on Image Processing 10, $1069-1080$.

Boehm, W., 1988. On de Boor-like algorithms and blossoming. Computer Aided Geometric Design 5, 71-79.

Böhm, W., Farin, G., Kahmann, J., 1984. A survey of curve and surface methods in CAGD. Computer Aided Geometric Design 1, 1-60.

Cavaretta, A., Dahmen, W., Micchelli, C., 1991. Stationary Subdivision, vol. 93. American Mathematical Society.

Conti, C., Romani, L., 2010. Affine combination of B-spline subdivision masks and its non-stationary counterparts. BIT Numerical Mathematics 50, $269-299$.

Conti, C., Gemignani, L., Romani, L., 2011. From approximating to interpolatory non-stationary subdivision schemes with the same generation properties. Advances in Computational Mathematics 35, 217-241.

Costantini, P., 1988. An algorithm for computing shape-preserving interpolating splines of arbitrary degree. Journal of Computational and Applied Mathematics 22, 89-136.

de Boor, C., DeVore, R., 1985. Partitions of unity and approximation. Proceedings of the American Mathematical Society 93, 705-709.

de Boor, C., Ron, A., 1992. The exponentials in the span of the multiinteger translates of a compactly supported function: Quasiinterpolation and approximation order. Journal of the London Mathematical Society 2, 519-535.

de Boor, C., DeVore, R.A., Ron, A., 1993. On the construction of multivariate (pre)wavelets. Constructive Approximation 9, 123-166.

Delgado-Gonzalo, R., Thévenaz, P., Seelamantula, C., Unser, M., 2011. Snakes with ellipse-reproducing properties. IEEE Transactions on Image Processing, in press, doi:10.1109/TIP.2011.2169975.

Deng, C., Wang, G., 2010. Incenter subdivision scheme for curve interpolation. Computer Aided Geometric Design 27, 48-59.

Derfel, G., Dyn, N., Levin, D., 1995. Generalized refinement equations and subdivision processes. Journal of Approximation Theory 80, $272-297$. 
Deslauriers, G., Dubuc, S., 1991. Fractals, Dimensions Non Entières et Applications, second ed. Masson, Paris. pp. 44-55.

Dyn, N., 1992. Subdivision schemes in computer aided geometric design. In: Light, W. (Ed.), Advances in Numerical Analysis, vol. 2. Clarendon Press, Oxford, New York, pp. 36-104.

Dyn, N., Levin, D., 1995. Analysis of asymptotically equivalent binary subdivision schemes. Journal of Mathematical Analysis and Applications 193, 594-621. Dyn, N., Gregory, J., Levin, D., 1991. Analysis of uniform binary subdivision schemes for curve design. Constructive Approximation 7, $127-147$.

Dyn, N., Levin, D., Luzzatto, A., 2008. Exponentials reproducing subdivision schemes. Foundations of Computational Mathematics 3, $187-206$.

Farin, G., 1997. Curves and Surfaces for Computer-Aided Geometric Design: A Practical Guide, fourth ed. Academic Press.

Farin, G., Rein, G., Sapidis, N., Worsey, A., 1987. Fairing cubic B-spline curves. Computer Aided Geometric Design 4, 91-103.

Jacob, M., Blu, T., Unser, M., 2002. Sampling of periodic signals: A quantitative error analysis. IEEE Transactions on Signal Processing 50, $1153-1159$.

Khalidov, I., Unser, M., 2006. From differential equations to the construction of new wavelet-like bases. IEEE Transactions on Signal Processing 54, 12561267.

Micchelli, C., Prautzsch, H., 1989. Uniform refinement of curves. Linear Algebra and its Applications 114-115, 841-870.

Pham, B., 1992. Offset curves and surfaces: A brief survey. Computer-Aided Design 24, 223-229.

Piegl, L., 1991. On NURBS: a survey. IEEE Computer Graphics and Applications 11, 55-71.

Romani, L., 2009. From approximating subdivision schemes for exponential splines to high-performance interpolating algorithms. Journal of Computational and Applied Mathematics 224, 383-396.

Romani, L., 2010. A circle-preserving C2 Hermite interpolatory subdivision scheme with tension control. Computer Aided Geometric Design 27, 36-47.

Ron, A., 1990. Factorization theorems for univariate splines on regular grids. Israel Journal of Mathematics 70, 48-68.

Sabin, M., Dodgson, N., 2004. A circle-preserving variant of the four-point subdivision scheme. In: Mathematical Methods for Curves and Surfaces. Tromsø, Norway. Nashboro Press, Brentwood, TN, pp. 275-286.

Schoenberg, I., 1964. On trigonometric spline interpolation. Indiana University Mathematics Journal 13, $795-825$.

Schumaker, L., 2007. Spline Functions: Basic Theory, third ed. Cambridge University Press.

Schwarz, L., 1966. Théorie des distributions. Hermann, Paris.

Späth, H., 1969. Exponential spline interpolation. Computing 4, 225-233.

Strang, G., Fix, G., 1971. A Fourier analysis of the finite element variational method. In: Constructive Aspect of Functional Analysis. Edizioni Cremonese, Rome, Italy, pp. 796-830.

Sunita, D., Shunmugaraj, P., 2009. An approximating C2 non-stationary subdivision scheme. Computer Aided Geometric Design 26 , $810-821$.

Unser, M., 2000. Sampling-50 years after Shannon. Proceedings of the IEEE 88, 569-587.

Unser, M., Blu, T., 2003. Wavelet theory demystified. IEEE Transactions on Signal Processing 51, 470-483.

Unser, M., Blu, T., 2005. Cardinal exponential splines: Part I-Theory and filtering algorithms. IEEE Transactions on Signal Processing 53, $1425-1438$.

Von Seggern, D., 1993. CRC Standard Curves and Surfaces. CRC Press.

Vonesch, C., Blu, T., Unser, M., 2007. Generalized Daubechies wavelet families. IEEE Transactions on Signal Processing 55, 4415-4429.

Warren, J., Weimer, H., 2002. Subdivision Methods for Geometric Design: A Constructive Approach, first ed. Morgan Kaufmann.

Zahn, C., Roskies, R., 1972. Fourier descriptors for plane closed curves. IEEE Transactions on Computers C-21, $269-281$.

Zhang, J., 1996. C-curves: An extension of cubic curves. Computer Aided Geometric Design 13, 199-217.

Zhang, J., Krause, F.-L., 2005. Extending cubic uniform B-splines by unified trigonometric and hyperbolic basis. Graphical Models 67, $100-119$. 